\title{
Earthquake nucleation on (aging) rate and state faults
}

\author{
A. M. Rubin and J.-P. Ampuero ${ }^{1}$ \\ Department of Geosciences, Princeton University, Princeton, New Jersey, USA \\ Received 11 February 2005; revised 3 August 2005; accepted 31 August 2005; published 29 November 2005.
}

[1] We obtain quasi-static, two-dimensional solutions for earthquake nucleation on faults obeying Dieterich's "aging" version of the rate and state friction equations. Two distinct nucleation regimes are found, separated by roughly $a / b \sim 0.5$, where $a$ and $b$ are the constitutive parameters relating changes in slip rate $V$ and state $\theta$ to frictional strength. When fault healing is unimportant $\left(V \theta / D_{c} \gg 1\right.$, where $D_{c}$ is the characteristic slip distance for the evolution of $\theta$ ), the nucleation zone spontaneously evolves toward a state of accelerating slip on a patch of fixed half length $L_{v} \approx 1.3774\left(\mu^{\prime} D_{c} / b \sigma\right)$, where $\mu^{\prime}$ is the intrinsic stiffness of the medium and $\sigma$ is the normal stress. This is the fixed length solution for which the stress intensity factor $K=0$. Although this solution does not depend upon $a / b$ explicitly, only for $a / b<0.3781$ does healing remain unimportant as instability is approached. For $a / b \gtrsim 0.5$ and a wide range of slow loading conditions, $V \theta / D_{c}$ ultimately approaches a quasi-constant value near 1 , and the nucleation zone takes on the appearance of an expanding slip-weakening crack. A fracture energy balance indicates that in this regime the nucleation length asymptotically approaches $\pi^{-1}[b /(b-a)]^{2}\left(\mu^{\prime} D_{c} / b \sigma\right)$, a result that is consistent with the numerical simulations despite considerable complexity as $a$ approaches $b$. This suggests that nucleation lengths can sometimes be much larger than those found by Dieterich (e.g., by a factor of 100 for $a / b=0.95$ ). For surfaces this close to velocity neutral, nucleation might produce signals detectable by surface seismometers for values of $D_{c}$ at the upper end of the lab range $(100 \mu \mathrm{m})$. However, the attributes of the aging law that give rise to such large nucleation lengths may be nonphysical; additional laboratory experiments are needed to address this issue.

Citation: Rubin, A. M., and J.-P. Ampuero (2005), Earthquake nucleation on (aging) rate and state faults, J. Geophys. Res., 110, B11312, doi:10.1029/2005JB003686.

\section{Introduction}

[2] Laboratory studies by Dieterich and others have shown that frictional strength depends upon the "state" of the sliding surface as well as the sliding velocity [Dieterich, 1979; Tullis, 1988; Marone, 1998]. A standard form of the constitutive law is

$$
\frac{\tau}{\sigma}=f^{*}+a \ln \frac{V}{V^{*}}+b \ln \frac{V^{*} \theta}{D_{c}}
$$

where $\tau$ is the frictional strength, $\sigma$ is the normal stress, $V$ is the sliding velocity, $\theta$ is the state variable (with units of time), $D_{c}$ is a characteristic sliding distance for the evolution of $\theta$ (of order $10 \mu \mathrm{m}$ for bare rock surfaces in the laboratory but up to $100 \mu \mathrm{m}$ for gouge), $f^{*}$ and $V^{*}$ are reference values of the friction and velocity, and $a$ and $b$ are both positive and of order $10^{-2}$. The parameter $a$ characterizes the

\footnotetext{
${ }^{1}$ Now at Institute of Geophysics, Seismology and Geodynamics, ETH-Honggerberg, Zurich, Switzerland.

Copyright 2005 by the American Geophysical Union. 0148-0227/05/2005JB003686\$09.00
}

magnitude of the "direct effect", the increase in strength with increasing sliding velocity (at constant state), and $b$ characterizes the magnitude of the "evolution effect", thought to reflect the increase in strength with increasing total area and/or cohesiveness of points of contact.

[3] Assuming nonzero sliding velocities, equilibrium dictates that the frictional strength equal the fault stress, which we partition into a boundary condition $\tau^{\infty}$ and an elastic component $\tau^{e l}$ due to nonuniform fault slip. Because most of the nucleation process takes place while inertial effects are unimportant, we focus on the quasi-static limit here. In two dimensions the static elastic stresses are proportional to the Hilbert transform $\mathcal{H}$ of the along-fault gradients in slip $\delta$ :

$$
\tau(x)=\tau^{\infty}(x)+\tau^{e l}(x)=\tau^{\infty}(x)+\frac{\mu^{\prime}}{2} \mathcal{H}\left[\delta^{\prime}\right](x),
$$

where

$$
\mathcal{H}\left[\delta^{\prime}\right](x) \equiv \frac{1}{\pi} \int_{-\infty}^{\infty} \frac{d \delta / d s}{s-x} d s
$$

In (2), $\mu^{\prime}$ represents the elastic shear modulus for antiplane deformation and the shear modulus divided by one minus Poisson's ratio for plane strain deformation, and $\delta^{\prime}=d \delta / d x$. 
[4] In contrast to the direct effect $a\left(\ln \left[V / V^{*}\right]\right)$, which appears to have a plausible basis in terms of a thermally activated Arrhenius process [Rice et al., 2003], laws for the evolution of state are essentially empirical. In this study we adopt Dieterich's "aging" law, which accounts for the laboratory observation that a fault surface strengthens or "heals" during stationary contact [Beeler et al., 1994; Dieterich and Kilgore, 1994; Marone, 1998]. With dots denoting time derivatives, this is written

$$
\dot{\theta}=1-\frac{V \theta}{D_{c}} .
$$

The first term on the right embodies the healing and shows that it accrues as elapsed time. The $V \theta / D_{c}$ term represents the weakening rate due to slip, or, as the healing rate is unity, the ratio of the weakening to healing rates. Because this quantity plays such a prominent role in this paper, we define the notation

$$
\Omega \equiv \frac{V \theta}{D_{c}} .
$$

When $\Omega=1$ the fault is at steady state $(\dot{\theta}=0)$. Following a step change in sliding velocity, $\theta$ evolves to a new steady state over the characteristic slip distance $D_{c}$.

[5] In equation (1), $f^{*}$ and $V^{*}$ are defined such that $\tau=$ $\sigma f^{*}$ when sliding at steady state with $V=V^{*}$, so that at steady state, (1) reduces to

$$
\tau=\sigma\left(f^{*}+[a-b] \ln \frac{V}{V^{*}}\right) .
$$

For $a<b$ the surface weakens with increasing sliding velocity (at steady state) and is prone to instability; for $a>b$ the surface is steady state velocity strengthening and sliding is stable. For temperatures and pressures appropriate for the seismogenic crust rock tends to be close to velocity neutral, to the extent that different experimental configurations sometimes lead to opposing determinations of the sign of $(a-b)$ even for loading conditions that are nominally the same [Marone, 1998]. Even considering only those experiments which show velocity-weakening behavior, a good median value of $a / b$ for granite is 0.9 (both for bare surfaces under a wide range of normal stress and slip speed [Kilgore et al., 1993], and for wet or nominally dry gouge under a wide range of temperature [Blanpied et al., 1998]).

[6] Despite two decades of interest in equations (1) and (3), their implications for the size of the nucleation zone on elastically deformable faults remains unclear. From a linear stability analysis, Ruina [1983] found the critical stiffness (the maximum permitting instability) of a spring-block slider to be $\sigma(b-a) / D_{c}$. Because stiffness in an elastic medium is roughly inversely proportional to size, this would seem to imply a nucleation length (or at least a minimum possible earthquake size) that scales roughly as $\mu^{\prime} D_{c} /(b-a) \sigma[$ Rice, 1993]. On the other hand, in a numerical study of earthquake nucleation in an elastic medium, Dieterich [1992] found the nucleation length to scale as $\mu^{\prime} D_{c} / b \sigma$, a result that was later shown to be consistent with laboratory experiments on meter-scale blocks of rock [Dieterich and Kilgore, 1996]. Dieterich pointed out that as slip accelerates and nucleation is well underway, the sliding velocity may greatly exceed steady state $\left(V \gg D_{c} / \theta\right)$, in which case (3) reduces to

$$
\dot{\theta} \approx-\frac{V \theta}{D_{c}}=-\Omega .
$$

We refer to this approximation as the "no-healing" limit, and with it, Dieterich [1994] obtained analytical solutions supporting his observation of scaling as $b^{-1}$ rather than $(b-a)^{-1}$, as well as his well-known results for earthquake aftershock rates. However, since $\theta$ decreases as $V$ increases during the approach to instability (when $\Omega>1$ ), it is not immediately evident from (3) when (6) becomes valid. To complicate the matter further, Lapusta and Rice [2002] describe numerical results that are inconsistent with both the $b^{-1}$ and $(b-a)^{-1}$ scaling. As these length scales appear repeatedly in this paper, we introduce the notation

$$
L_{b} \equiv \frac{\mu^{\prime} D_{c}}{b \sigma} ; \quad L_{b-a} \equiv \frac{\mu^{\prime} D_{c}}{(b-a) \sigma} .
$$

Because large earthquakes and transient creep events often appear to nucleate near the base of the seismogenic zone, near the transition from velocity weakening to velocity strengthening where $L_{b}$ and $L_{b-a}$ diverge, understanding what controls the nucleation length under these conditions would seem to be important.

[7] For completeness, we state here the second common equation for the evolution of $\theta$, referred to as the "slip" law because no evolution occurs during stationary contact:

$$
\dot{\theta}=-\frac{V \theta}{D_{c}} \ln \frac{V \theta}{D_{c}}=-\Omega \ln \Omega .
$$

For small departures from steady state $(\Omega=1+\epsilon$, with $|\epsilon| \ll 1)$, (8) asymptotically approaches (3). In recent years the slip law seems to have fallen out of favor somewhat, probably because the aging law has been shown to be superior in the range $\Omega \ll 1$ where healing dominates [Beeler et al., 1994]. In hindsight, however, it appears to be the range $\Omega \sim 1$ to $\Omega \gg 1$ that controls the nucleation size. On the basis of the results to follow, we expect nucleation to be sometimes significantly different under the two laws. Further experimental and theoretical work to sort this out is certainly warranted.

[8] This paper is organized as follows. In section 2 we present numerical simulations that mimic those of Dieterich [1992], and illustrate two fundamentally different nucleation regimes distinguished by the behavior of $\Omega$. In the first regime $\Omega \gg 1$ everywhere, and nucleation is characterized by accelerating slip on a patch of fixed length. In the second regime $\Omega$ in the interior of the nucleation zone tends to a quasi-constant (and $a / b$-dependent) value of order 1 , a condition we refer to as the "constant-weakening" limit. Nucleation in this case takes the form of an expanding crack with time-varying slip-weakening properties.

[9] In section 3 we derive a separable solution for fixed length nucleation in the limit $\Omega \gg 1$. Requiring the stresses at the ends of the nucleation zone to remain finite fixes the 

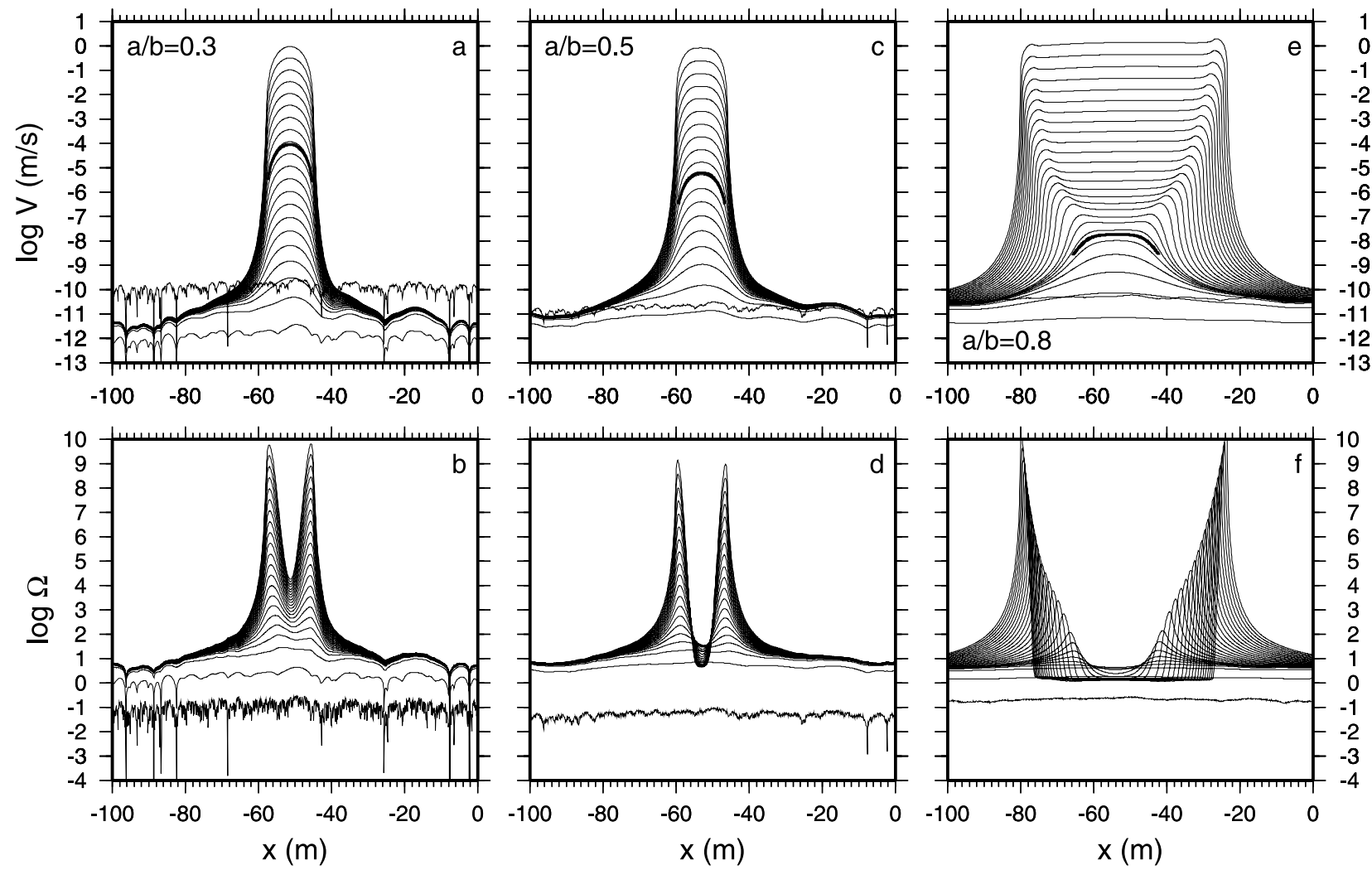

Figure 1. Snapshots of (a, c, e) velocity and (b, d, f) $\Omega \equiv V \theta / D_{c}$ for a fault with heterogeneous initial conditions and $a / b=0.3$ (Figures $1 \mathrm{a}$ and $1 \mathrm{~b}$ ), 0.5 (Figures $1 \mathrm{c}$ and $1 \mathrm{~d}$ ), and 0.8 (Figures $1 \mathrm{e}$ and $1 \mathrm{f}$ ). The bold velocity profiles extend from stressing rate peak to stressing rate peak when that distance reaches its minimum value $\left(2 L_{\mathrm{min}}\right)$. In this and all subsequent figures, $\mu^{\prime}=11.56 \mathrm{GPa}, \sigma=100 \mathrm{MPa}, D_{c}=0.4 \mathrm{~mm}$, and (unless noted otherwise) $b=0.01$.

nucleation (half)length to be $1.3774 L_{b}$, for practical purposes identical to the value identified by Dieterich [1992] on the basis of numerical simulations. Within this solution, however, only for $a / b<0.3781$ does $\Omega$ increase during the approach to instability. For larger $a / b, \Omega$ decreases and for our adopted loading conditions the Dieterich approximation becomes invalid before elastodynamic speeds are reached. Relaxing the finite stress constraint, we find that for $a / b<$ 0.3781 instabilities can nucleate within regions smaller than $1.3774 L_{b}$. Such solutions may be relevant for fault segments bounded by strong barriers, and provide direct evidence that nucleation zones need not evolve to the shortest length permitting instability. For $a / b=0$ this shortest length coincides with the value $0.579 L_{b}$ found for linear slipweakening friction by Uenishi and Rice [2003].

[10] In section 4 we explore nucleation in the constantweakening regime. A fracture energy balance shows that in the limit of large slip speeds the nucleation half length approaches $\pi^{-1}[b /(b-a)]^{2} L_{b}$. This estimate is well supported by the simulations, although how closely the nucleation length approaches this value by the time dynamic slip speeds are reached depends upon the loading conditions. We close this section by examining some implications for the detectability of seismic nucleation phases, and discuss the (perhaps suspect) aspects of the aging law that give rise to such large nucleation zones for $a$ near $b$.

[11] The fixed length solution essentially guarantees that nucleation for low $a / b$ occurs in the no-healing regime, but whether nucleation for larger $a / b$ occurs in the constantweakening regime depends also upon the loading conditions. Both regimes represent mature phases of nucleation in which the region of accelerating slip is already highly localized. They are preceded by an earlier localization phase characterized by an increasing $\Omega$. If the value of $\Omega$ upon localization is large enough, then over some velocity range the no-healing solution is followed even for large $a / b$. In section 5 and related appendices we derive analytic results that are useful for assessing qualitatively the evolution of $\Omega$ during these earliest stages of nucleation, and the related (and perhaps more general) question of what determines whether the nucleation zone expands or contracts.

\section{Quasi-Static Numerical Simulations}

[12] To solve the governing equations numerically, we equate (1) and (2) and differentiate with respect to time to obtain

$$
a \frac{\dot{V}}{V}+b \frac{\dot{\theta}}{\theta}=\frac{\dot{\tau}^{\infty}}{\sigma}+\frac{\mu^{\prime}}{2 \sigma} \mathcal{H}\left[V^{\prime}\right] .
$$

The parameters $\sigma, a$, and $b$ are taken to be constant and uniform, while $V, \theta$, and perhaps $\dot{\tau}^{\infty}$ vary in space and time. We compute the elastic stresses from the velocity gradients in the spectral domain, using the result that for each wave number $\kappa$ the stressing rate is related to the velocity $V_{\kappa}$ by $\dot{\tau}_{\kappa}=-V_{\kappa} \mu^{\prime}|\kappa| / 2$. 
[13] Together, equations (9) and (3) allow one to compute $\dot{v}$ and $\dot{\theta}$ in terms of the known $\theta, V$ and $\dot{\tau}^{\infty}$ at the start of each time step. The change in $V$ and $\theta$ during a time step of duration $\Delta t$ are then $V \Delta t$ and $\dot{\theta} \Delta t$. We solve these equations on a grid of $2^{13}-2^{16}$ points along the fault, with a uniform spacing of $0.05-0.5 \mathrm{~m}$, using the adaptive time stepping Bulirsch-Stoer routine of Press et al. [1986].

[14] We first show results of simulations where the fault is initially healing $(\Omega<1)$. Because the fault strengthens as the logarithm of time, a remote stress that increases linearly with time ensures that the fault ultimately progresses to failure by passing through steady state $(\Omega=1)$ from below. In these simulations the initial velocity is $10^{-9} \mathrm{~m} / \mathrm{s}$, the remote stressing rate is $10^{-2} \mathrm{~Pa} / \mathrm{s}$, and the initial state is randomly distributed between 0 and $D_{c} / V$ (steady state). The nucleation zone first passes through steady state at a velocity of $\sim 10^{-12}-10^{-11} \mathrm{~m} / \mathrm{s}$. In Appendix A2 we examine the limit cycle oscillations of a spring-block slider with radiation damping, and estimate the velocity at which a "locked" fault crosses the steady state curve to be a few orders of magnitude less than the driving plate velocity. Thus we believe that, at least in a very gross sense, our simulations generate an appropriate range of velocities between the initial crossing of the steady state curve and elastodynamic speeds.

[15] Figure 1 shows the evolution of both $V$ and $\Omega$ for $b=$ 0.01 and three values of $a / b$, and illustrates the main styles of nucleation we observe. In the upper panels, the velocity initially decreases but increases between the second and third snapshots. Larger values of $a$ lead to more rapid smoothing of the initial heterogeneity through the direct velocity effect (increased frictional resistance where the velocity is largest). This is also consistent with the linear stability analysis of Rice and Riuna [1983], which shows that variations on length scales shorter than $L_{b-a}$ are damped. For $a / b=0.3$, the instability appears to grow in amplitude while maintaining a fixed shape. For $a / b=0.5$, the instability appears initially to maintain that same shape, but eventually the velocity profile flattens in the center. We show in section 3 that this distinction from $a / b=0.3$ is not an artifact of the number of time steps taken. For $a / b=0.8$ the nucleation zone first localizes and then expands in a crack-like fashion (peak velocities near the tips; relatively uniform velocities in the interior). In the lower panels, $\Omega$ first decreases and then increases between the second and third snapshots. Near the center of the instability, $\Omega$ increases monotonicallly for $a / b=0.3$, but eventually decreases to a quasi-constant and quasi-uniform value near 1 for $a / b=0.5$ and 0.8 .

[16] In their study of earthquake nucleation on a slipweakening fault, Uenishi and Rice [2003] found that in the presence of a peaked and uniformly increasing load, the slipping region expands continuously until a stable elastic configuration no longer exists. This largest stable size is an obvious choice for the definition of nucleation length for slip-weakening friction. For rate-and-state friction this definition is inappropriate, both because the entire surface is slipping and because it appears that the nucleation zone first shrinks via some localization process (this localization is most easily seen in snapshots of the stressing rate, an example of which is shown in Figure 4 in section 3). By analogy with crack expansion on an otherwise stationary

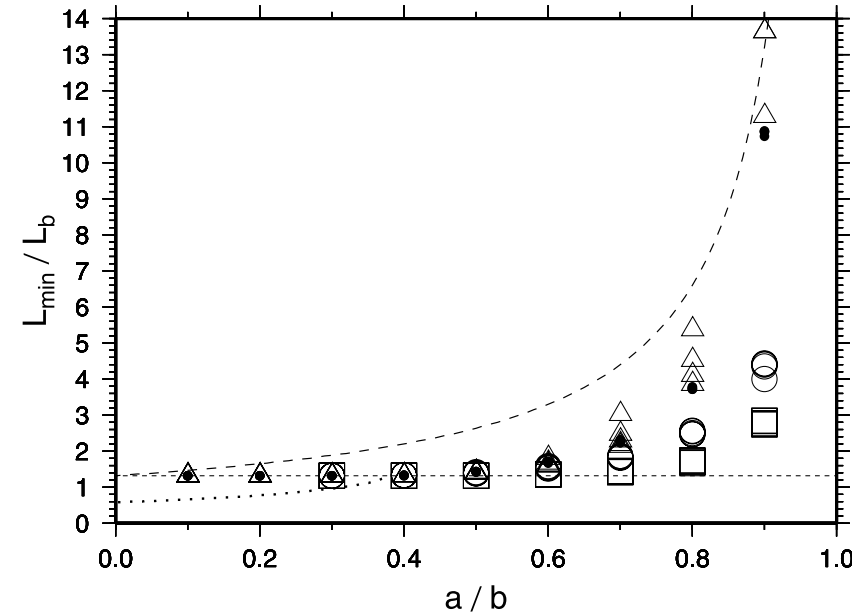

Figure 2. Plot of normalized $L_{\min }$, defined as half the minimum (time-dependent) distance between stressing rate peaks at opposite ends of the nucleation zone, for four suites of simulations with differing boundary/initial conditions and variable $b$ and $a / b$. The normalizing length scale $L_{b} \equiv \mu^{\prime} D_{c} /$ $b \sigma$. For $a / b=0.9$ and a few values of $b$ the algorithm for detecting the minimum length was unsuccessful; those results are not shown. The horizontal dashed line shows the length equal to $95.83 \%$ of that given by equation (21). The dotted curve below that shows the estimate from equation (26) of the shortest nucleation zone capable of reaching instability via the no-healing, fixed length solution of section 3. The dashed curve shows scaling of the nucleation length as $(b-a)^{-1}$, arbitrarily scaled to the simulation results for $a=0$, and is drawn only for reference.

surface, we adopt as a quantitative measure of nucleation length half the distance between the peaks in elastic stressing rate to either side of the nucleating patch. We define $L_{\min }$ to be the first minimum of the time-dependent nucleation length following the localization process. The bold curves in Figure 1 show the velocity profiles at this minimum length, drawn from stressing rate peak to stressing rate peak, so their length is $2 L_{\mathrm{min}}$.

[17] The values of $L_{\min }$ for several dozen simulations are summarized in Figure 2. For each set of loading conditions, simulations were carried out for 5 values of $b$ (in equal increments from 0.005 to 0.025 ) and 9 values of $a / b$. The nucleation lengths are normalized by $L_{b}$. The dimensionless versions of the governing equations (Appendix A1) show that, with this scaling, varying $b$ at a constant $a / b$ is equivalent to varying either the initial values of both $V$ and $\theta^{-1}$, or $\sigma / \dot{\tau}^{\infty}$, by the same factor. The set of simulations from which the panels of Figure 1 were extracted are shown by open circles. The squares are for the same conditions except that the initial state was randomly distributed between 0.495 and 0.505 times $D_{c} / V$, rather than between 0 and $D_{c} / V$. The small solid circles are for a homogeneous fault undergoing steady state sliding $(\Omega=1)$ that is then subjected to a remote loading rate restricted to a region that is small compared to $L_{\min }\left(0.1 L_{b-a}\right)$. The triangles are for a fault on which $\Omega$ peaks at 1 at the origin and decreases smoothly to $10^{-1 / 2}$ at a distance of $2 L_{b-a}$ to either side, subjected to a uniform remote stressing rate of $10^{-3} \mathrm{~Pa} / \mathrm{s}$. 


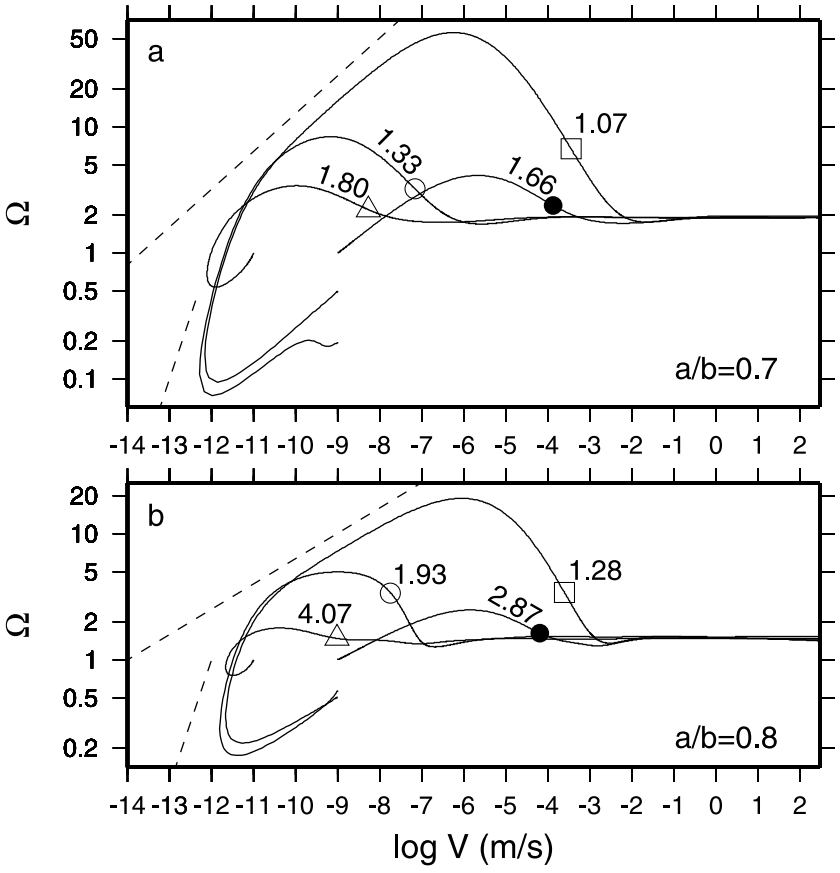

Figure 3. Evolution of $\Omega$ as a function of $V$, evaluated at the center of the nucleation zone, for $a / b=$ (a) 0.7 and (b) 0.8 . The four simulations with $b=0.01$ are shown. The symbols correspond to the different loading conditions as in Figure 2 and indicate when $L_{\min }$ is reached. The numbers give the ratio of the measured $L_{\min }$ to that for small $a / b$ (horizontal dashed line in Figure 2); this ratio approaches 1 as the peak value of $\Omega$ increases. Also evident is the tendency to evolve to a quasi-constant value of $\Omega$ that decreases with increasing $a / b$, independent of the loading conditions. The top dashed line in Figures $3 \mathrm{a}$ and $3 \mathrm{~b}$ shows the slope of the trajectory for zero stress change (equation (51)), and the bottom dashed line the slope for constant state.

[18] For $a / b \leq 0.4$, the $L_{\min }$ for the various $b$ collapse to the same normalized value of $\sim 1.320$, in almost all cases to within $10 \%$ of the discretization interval (the locations of the stressing rate peaks are interpolated). This agreement is all the more significant because for the randomized initial conditions the position of the nucleating patch along the fault varies with $b$, even at the same $a / b$. These results indicate scaling as $b^{-1}$ and independence of $a$, as found by Dieterich [1992]. For larger $a / b$ there is significant dependence upon $a$, and for a given $a / b$ there is more scatter both between the different sets of loading conditions (different symbols) and within a single set (different values of $b$ ). The dashed curve indicates scaling as $(b-a)^{-1}$, adjusted to equal 1.32 at $a=0$. All the simulations fall between this curve and 1.32 , and they consistently approach the low $a / b$ value more closely as the maximum value of $\Omega$ reached during the earlier stages of nucleation becomes larger (Figure 3).

\section{No-Healing Regime: Localized Acceleration}

[19] The velocity profiles in Figure 1a are suggestive of accelerating slip on a fixed length patch with a time- invariant velocity distribution. Here we treat this patch as a crack. The right panels suggest that time invariance requires the no-healing limit $(\Omega \gg 1)$, in which case one can substitute (6) for $\dot{\theta}$ into (9) to eliminate the state variable from the equilibrium equation:

$$
a \frac{\dot{V}}{V}-b \frac{V}{D_{c}}=\frac{\dot{\tau}^{\infty}}{\sigma}+\frac{\mu^{\prime}}{2 \sigma} \mathcal{H}\left[V^{\prime}\right]
$$

[20] We seek a separable solution of the form

$$
\begin{gathered}
V(x, t)=V_{o}(t) \mathcal{V}(x / L), \quad|x|<L ; \\
V(x, t)=0, \quad|x| \geq L,
\end{gathered}
$$

where $L$ is the fixed effective crack length and the velocity distribution $\mathcal{V}$ is a function of dimensionless position $x / L$, normalized such that $\mathcal{V}(0) \equiv 1$. We expect this $V$ to satisfy (10) for $|x|<L$ but not $|x| \geq L$ (except in the sense that the velocities satisfying (10) for $|x|>L$ should be negligible compared to those for $|x|<L-\epsilon$, for $\epsilon \ll L)$. The corresponding elastic stressing rate is

$$
\dot{\tau}^{e l}(x, t)=V_{o}(t) \frac{\mu^{\prime}}{2 L} \mathcal{T}(x / L) .
$$

Writing $\xi$ for $x / L$ and $\mathcal{V}^{\prime}$ for $d \mathcal{V} / d \xi$, the dimensionless stressing rate distribution $\mathcal{T}(\xi)$ equals $\left(L / V_{o}\right) \mathcal{H}\left[\mathcal{V}^{\prime}\right]$ (note that $\mathcal{T}(0)<0)$. In the following we refer to this as the "fixed length" solution; constancy of the normalized velocity distribution is implicit.

[21] For a locked fault the remote stressing rate $\dot{\tau}^{\infty}$ should be of order $\mu^{\prime} V_{p l} / l$, where $V_{p l}$ is the plate velocity and $l$ is a representative length scale for strain accumulation (e.g., the depth to the freely slipping zone on a vertical strike-slip fault, or perhaps a small fraction of this value if the nucleation zone lies near the locked/creeping transition). The elastic stressing rate is of order $\mu^{\prime} V_{o} / L$. Thus the criterion for neglecting $\dot{\tau}^{\infty}$ in (10) is $V_{o} \gg V_{p l}(L / l)$. In seeking the separable solution we have already assumed $V_{o} \gg V_{x>L}$, so unless the slip velocities outside the nucleating patch are orders of magnitude less than the plate velocity (perhaps 3-4 orders less), $V_{o} \gg V_{x>L}$ is a more stringent assumption than $V_{o} \gg V_{p l}(L / l)$ and we neglect $\dot{\tau}^{\infty}$ in (10).

[22] Substituting (11), (13), and $\dot{\tau}^{\infty}=0$ into (10) and rearranging yields

$$
\frac{\dot{V}_{o}}{V_{o}^{2}}=\frac{b}{a D_{c}}\left[\mathcal{V}+\frac{L_{b}}{2 L} \mathcal{T}\right]
$$

For a function of time (on the left) to equal a function of position (on the right), both must be constant. Evaluating (14) at $x=0$ yields

$$
\frac{\dot{V}_{o}}{V_{o}^{2}}=\frac{b}{a D_{c}}\left[1+\frac{L_{b}}{2 L} \mathcal{T}(0)\right] \equiv \frac{C_{1}}{D_{c}}
$$



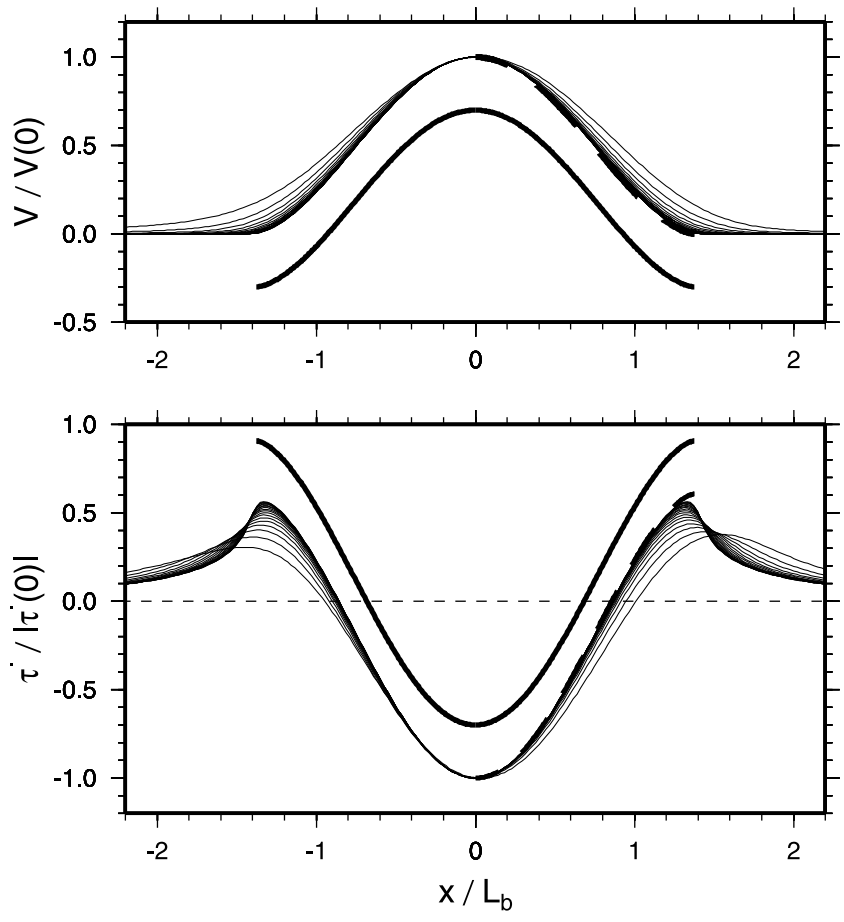

Figure 4. (top) The last 17 of the 21 velocity snapshots in Figure 1a, normalized by their peak magnitude and shifted to the origin, with distances scaled by $L_{b}=4.622 \mathrm{~m}$. The profiles asymptotically approach the fixed length solution for which $K=0$, as shown by the bold dashed curve for $x>$ 0 and the bold solid curve for all $x$ (the latter offset vertically for clarity). (bottom) The corresponding stressing rate profiles are shown, again normalized by peak magnitude and with the $K=0$ fixed length solution superimposed.

with solution

$$
V_{o}=V_{o}(0)\left(1-t / t^{*}\right)^{-1} ; \quad t^{*} \equiv C_{1}^{-1} D_{c} / V_{o}(0),
$$

where, for $C_{1}>0, t^{*}$ is the time of instability. That the slip velocity increases as the inverse of the time to instability is a general property of single-degree-of-freedom systems obeying equation (10) with $\dot{\tau}$ proportional to $V$. For a spring-block slider with stiffness $k$ and negligible remote loading rate,

$$
C_{1}=\frac{b}{a}\left[1-\frac{k D_{c}}{b \sigma}\right]
$$

Thus a nucleating patch following the fixed length solution can be thought of as a single-degree-of-freedom system with an "effective stiffness" at the origin of $k(0)=-\left(\mu^{\prime} /\right.$ $2 L) \mathcal{T}(0)$, a result that follows immediately from (13) [see also Dieterich, 1992, equation (25)].

[23] Subtracting (15) from (14) yields the following relation between the normalized velocity and stressing rate distributions:

$$
\mathcal{V}(\xi)=1-\frac{L_{b}}{2 L}[\mathcal{T}(\xi)-\mathcal{T}(0)]
$$

Given any value of the intrinsic length scale $L_{b}$, equation (18) enables one to compute $\mathcal{V}$ and $\mathcal{T}$ for any $L$. However, requiring that the nucleation zone neither expand nor contract also requires that the stresses at the tip of the corresponding "effective crack" be finite (that is, that the stress intensity factor $K$ at the tip equal zero), and this fixes $L$ to a unique value, which we write as $L_{\nu}$. An equation analogous to (18) with the $K=0$ constraint also arises in the context of a uniformly stressed slip-weakening crack in the large-scale yielding regime [Chen and Knopoff, 1986]. Note that because (18) is independent of $a$, so too are $\mathcal{V}, \mathcal{T}$, and $L_{\nu} ; a$ controls the time of instability through equations (15) and (16).

[24] To solve (18) numerically, the Hilbert transform of the velocity gradients can be inverted to determine $\mathcal{V}$ in terms of the stressing rate (symmetric about the origin)[e.g., Spence and Sharp, 1895]:

$$
\mathcal{V}(\xi)=-\frac{2}{\pi \mu^{\prime}} \int_{0}^{1} \ln \left|\frac{\sqrt{1-\xi^{2}}-\sqrt{1-s^{2}}}{\sqrt{1-\xi^{2}}+\sqrt{1-s^{2}}}\right| \mathcal{T}(s) d s
$$

If $\mathcal{T}$ is approximated as piecewise linear over $M$ segments along the half crack then the integral can be evaluated analytically. Assuming a continuous stress distribution leads to $M+1$ unknown stress values, which we determine by satisfying (18) at the center of each segment as well as at the origin (where $\mathcal{V} \equiv 1$ ). A normalized $K$ is determined analytically from the same symmetric, continuous, and piecewise linear stress distribution using [Lawn, 1993]

$$
K=\frac{2}{\pi} L^{1 / 2} \int_{0}^{1} \frac{\mathcal{T}(\xi)}{\sqrt{1-\xi^{2}}} d \xi
$$

[25] Consistent with the results of Chen and Knopoff [1986], we find by iteration that enforcing $K=0$ requires $L_{b} / L=0.7260$, or

$$
L_{\nu}=1.3774 L_{b}
$$

(10\% larger than the value of $1.25 L_{b}$ proposed by Dieterich [1992]), and fixes $\mathcal{T}(0)=-1.7132$ (compared to -1 for a uniform stress drop). For these values the constant $C_{1}$ in (15) is indeed positive, as is required for instability:

$$
C_{1}=0.3781 \frac{b}{a}
$$

The stress distribution satisfying (18) for smaller $L$ yields $K>0$, and if imposed as an initial condition would cause the effective crack to grow, while the stress distribution for larger $L$ yields $K<0$ (the direction of slip near the crack ends is opposite to that at the center), which would require a change in sign of the ambient stress.

[26] In Figure 4 we compare the distributions of $\mathcal{V}$ and $\mathcal{T}$ yielding $K=0$ to the simulations for $a / b=0.3$ in Figure 1 . The 17 snapshots with a peak velocity above $10^{-9} \mathrm{~m} / \mathrm{s}$ are shown after normalization by their peak magnitudes. For the adopted parameter values, $L_{\nu}=6.366 \mathrm{~m}$. Both the velocity (top) and stressing rate (bottom) profiles asymptotically approach the fixed length distributions. The only apparent difference at the time of the last snapshot is in the stressing 


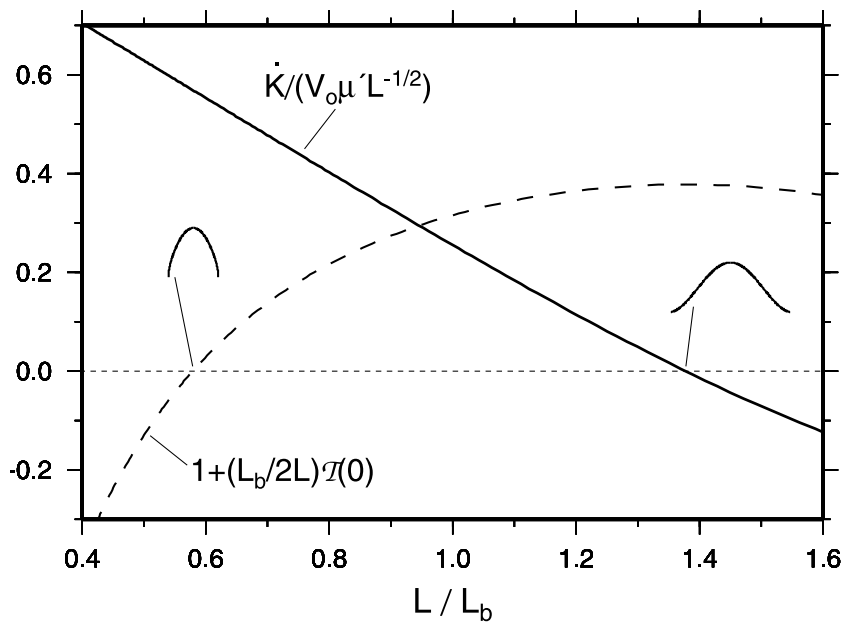

Figure 5. Plot of the dimensionless stress intensity factor rate, $\dot{K} /\left(V_{o} \mu^{\prime} / L^{1 / 2}\right)$ (solid curve), and $1+\left(L_{b} / 2 L\right) \mathcal{T}(0)$ (dashed curve), as a function of dimensionless crack length $L / L_{b}$. The dashed curve is equivalent to $k(0)=(b-a) \sigma / D_{c}$, where $k(0)$ is the effective stiffness at the origin, and defines the shortest viable nucleation zone for $a / b<0.3781$ in Figure 2. Also shown are scaled velocity profiles for $L / L_{b}=$ $1.3774(K=0)$ and $0.579\left(k(0)=b \sigma / D_{c}\right)$.

rate at the crack tips, which are well defined in the fixed length solution but not in the numerical simulation. Related to this, perhaps, is a very consistent $4.35 \%$ difference between the crack length given by (21) and the (smaller) distance between the stressing rate peaks in the numerical simulations that we do not consider further.

\subsection{Applicability of the No-Healing Limit}

[27] Assuming $\Omega \gg 1$ when the fixed length solution is first approached (as in Figures $1 \mathrm{~b}$ and 1d), we can substitute the velocity history from (11) and (16) into (6) for $\dot{\theta}$ to determine when the no-healing approximation remains valid. Integrating equation (6), multiplying by $V(\xi, t) / V(\xi$, 0 ), and making use of (16) yields

$$
\frac{\Omega(\xi, t)}{\Omega(\xi, 0)}=\left(\frac{V_{o}(t)}{V_{o}(0)}\right)^{1-\mathcal{V}(\xi) / C_{1}} .
$$

Thus $\Omega$ is an increasing function of velocity for $\mathcal{V}(\xi) / C_{1}<1$, and a decreasing function for $\mathcal{V}(\xi) / C_{1}>1$. Equation (6) remains a valid approximation until instability $(V=\infty)$ only for $\mathcal{V}(\xi) / C_{1} \leq 1$. Because $\mathcal{V}$ is maximal at the crack center and tends to zero at $x / L= \pm 1, \Omega$ continually increases near the crack ends and the no-healing approximation is first violated (if ever) at $x=0$ (Figure 1). Substituting $\mathcal{V}(0)=1$ and equation (22) for $C_{1}$ into (23), (6) remains valid until instability only for

$$
C_{1}>1 \Longrightarrow \frac{a}{b}<0.3781 .
$$

[28] This result explains many of the pertinent features of Figures 1 and 2. The fixed length solution places no restrictions on $a / b$, not even that $a<b$; it requires only that $\Omega \gg 1$. When equation (24) is satisfied, $\Omega$ increases monotonically and all our simulations evolve to a nucleation length equal to (96\% of) that given by (21). As (24) is progressively violated, $\Omega$ decreases more and more rapidly at the crack center (by 0.3 decades for each decade increase in $V$ for $a / b=0.5$, compared to 1.4 decades for each decade increase in $V$ for $a / b=0.9$ ). If $\Omega$ is sufficiently large as nucleation localizes, the fixed length solution may remain nearly valid over a velocity increase of many orders of magnitude even for $a / b>0.5$. This is clearly seen in our Figure 1c and in Figures 4 and 5 of Dieterich [1992]. The degree to which the fixed length solution accurately predicts the nucleation length for $a / b>0.3781$, and the variable $L_{\min }$ for $a / b \gtrsim 0.5$ in Figure 2 , presumably reflect how the loading conditions control $\Omega$ upon localization.

[29] We note that retaining a uniform and constant remote stressing rate leads to a more complicated equation for $V_{o}(t)$ analogous to equation (26) of Dieterich [1992]. This equation tends to our equation (16) provided $t=0$ is defined at a time when $\dot{\tau}^{\infty} \ll\left|\dot{\tau}^{e l}\right|$. A nonzero $\dot{\tau}^{\infty}$ does not influence the velocity distribution $\mathcal{V}$ because it drops out in the derivation of equation (18).

\subsection{Relation to Rate and State Spring-Block Sliders and Slip-Weakening Faults}

[30] Ranjith and Rice [2003] found that spring-block sliders obeying equations (1) and (3) could go unstable only for spring stiffnesses less than a critical value $k_{c r}=(b-$ a) $\sigma / D_{c}$, even for arbitrarily large perturbations from steady state (unstable in the sense that the quasi-static equations lead to infinite velocities). For an elastic continuum the effective stiffness should be nearly inversely proportional to $L$, so this result might seem to contradict the assertion that the nucleation length scales as $b^{-1}$ rather than (the apparently larger) $(b-a)^{-1}$ when (24) is satisfied. To explore this further, we can summarize from equations (15) and (24) the conditions for instability of the fixed length solution as

Instability in the limit $\Omega \gg 1\left(C_{1}>0\right)$

$$
1+\frac{L_{b}}{2 L} \mathcal{T}(0)>0
$$

$\Omega \gg 1$ until instability $\left(C_{1} \geq 1\right)$

$$
1+\frac{L_{b}}{2 L} \mathcal{T}(0) \geq \frac{a}{b}
$$

It is immediately apparent that for $a, b>0$ the second of these criteria is more stringent than the first. In terms of the effective stiffness at the crack center $k(0)=-\left(\mu^{\prime} / 2 L\right) \mathcal{T}(0)$, criteria (25) and (26) become respectively $k(0)<b \sigma / D_{c}$ and $k(0) \leq(b-a) \sigma / D_{c}$ (recall that $\left.L_{b} \equiv \mu^{\prime} D_{c} / b \sigma\right)$. Thus there is no inconsistency with the results of Ranjith and Rice. For the fixed length crack, the constraint $K=0$ gives rise to an effective stiffness that is low enough for $\Omega$ to increase until instability, provided $a / b$ is sufficiently small.

[31] It is worth emphasizing that the $K=0$ solution to which the simulations evolve is not the minimum length solution. To estimate the minimum size we can drop the $K=$ 0 constraint on the fixed length solution, relying instead on whatever is constraining the slipping region to prevent lateral growth. Figure 5 shows values of the normalized stress intensity factor and $1+\left(L_{b} / 2 L\right) \mathcal{T}(0)$ obtained by 

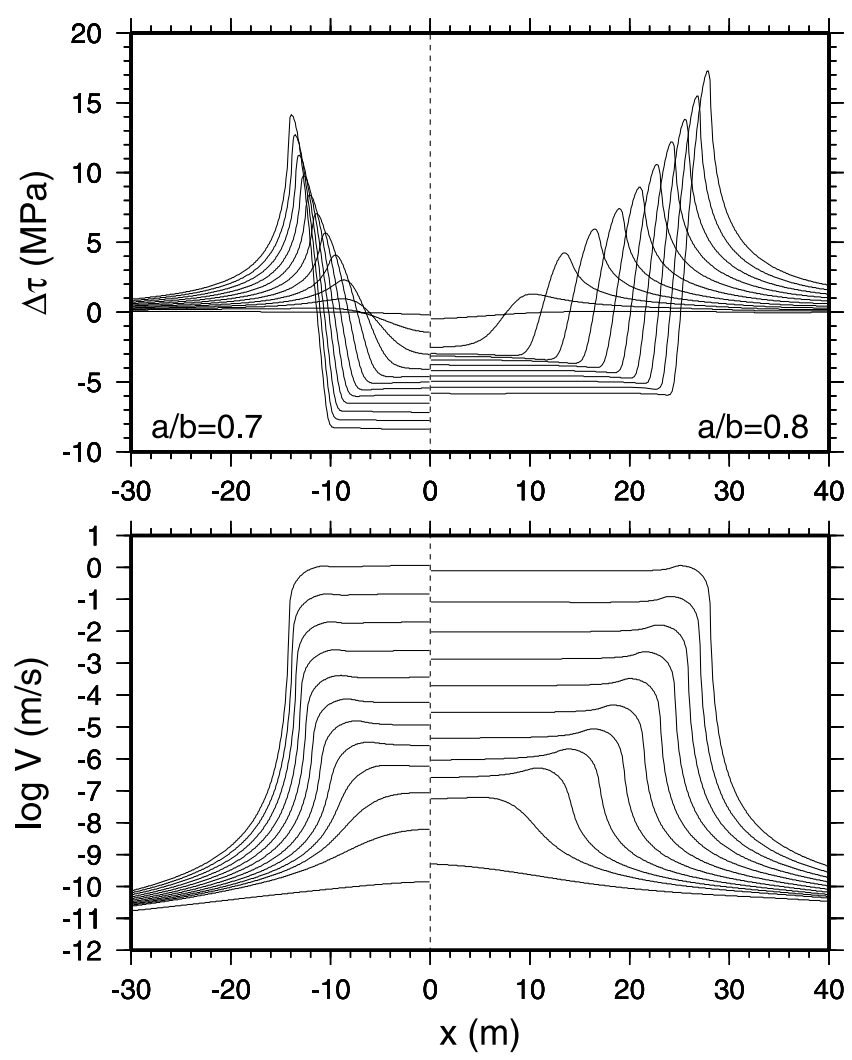

Figure 6. Snapshots of the change in (top) shear stress and (bottom) velocity for $a / b=0.7$ (left) and 0.8 (right), for simulations with heterogeneous initial state (Figure 1 and open circles in Figure 2). Stresses are relative to the average initial stress; distances are from the center of the nucleation zone.

solving (18) for a range of $L / L_{b}$. The zero crossing of the $1+$ $\left(L_{b} / 2 L\right) \mathcal{T}(0)$ curve indicates the minimum $L$ satisfying (25), and the value of that curve shows, for each $L$, the maximum value of $a / b$ satisfying (26). Thus along the $1+\left(L_{b} / 2 L\right) \mathcal{T}(0)$ curve, $k(0)=(b-a) \sigma / D_{c}$.

[32] This $k(0)=(b-a) \sigma / D_{c}$ curve is drawn as the dotted line in Figure 2. It indicates, as a function of $a / b$, the shortest fixed length nucleation zone satisfying (26). At $a=$ 0 this minimum length coincides with the universal nucleation length of $0.579 L_{b}$ found by Uenishi and Rice [2003] for linear slip-weakening behavior. In a sense this is comforting; for $a=0$ equation (10) reverts to pure slip weakening with weakening rate $W=-d \tau / d \delta=-\dot{\tau} / V=b \sigma / D_{c}$. Mathematically, for $1+\left(L_{b} / 2 L\right) \mathcal{T}(0)=0$ our equation (18) for $\mathcal{V}$ is identical to equation (12) of Uenishi and Rice for the velocity distribution of a nucleating crack poised at the stable/unstable transition. Physically, however, the situations in which these velocity distributions arise seem quite different: Acceleration of a pinned crack with $K>0$ in our case; the onset of unstable growth for a (previously) stably expanding crack satisfying $K=0$ in theirs. Our case is conceptually more similar, but still not identical, to that of Dascalu et al. [2000], who found the same minimum length for a fixed length elastodynamic crack with linear slip-weakening behavior, stressed uniformly at the peak strength.

[33] In a numerical study of rate-and-state friction, Lapusta and Rice [2002] found nucleation lengths that agreed with the slip-weakening $(a=0)$ estimate of Uenishi and Rice for $a<10^{-6}$ but that "more than doubled" for $a=$ $10^{-4}$. From Figure 2 it appears that this reflects a transition from the $1+\left(L_{b} / 2 L\right) \mathcal{T}(0)=0$ solution to the $K=0$ solution of equation (18). We found no nucleation lengths lying along the dotted curve in Figure 2; presumably this would require larger stress barriers (to pin the crack) than we simulated.

\section{Constant-Weakening Regime: Quasi-Static Crack Growth}

[34] For sufficiently large values of $a / b$, the fixed length solution is ultimately lost (or perhaps it was never attained) and the nucleation zone takes on the appearance of an expanding crack. Slip velocities, rather than being peaked at the center, are more nearly uniform. Stresses are also quite uniform except near the tips, where the distribution is suggestive of linear slip-weakening behavior (Figure 6). Concurrently, $\Omega$ in the interior of the nucleation zone decreases to a near-constant value modestly larger than 1 . This value decreases with increasing $a / b$ and for a given $a / b$ appears to be largely independent of the loading conditions (Figure 3). In this section we use these observations to develop an asymptotic expression for the nucleation length as a function of $a / b$.

[35] We first note that having a quasi-uniform stress drop and (log) velocity is consistent with equation (1) and elasticity. For a quasi-statically expanding crack with half length $L$ and a uniform (negative) stress drop $\Delta \tau$, the slip and slip rate are

$$
\begin{gathered}
\delta(x)=-\frac{2 L \Delta \tau}{\mu^{\prime}}\left(1-\frac{x^{2}}{L^{2}}\right)^{1 / 2}, \\
V(x)=-\frac{2}{\mu^{\prime}}\left[(\dot{\Delta \tau}) L\left(1-\frac{x^{2}}{L^{2}}\right)^{1 / 2}+\Delta \tau \dot{L}\left(1-\frac{x^{2}}{L^{2}}\right)^{-1 / 2}\right] .
\end{gathered}
$$

The first term within the brackets in (28) gives the elliptical distribution due to the changing stress on a crack of fixed length; the second term is the distribution from an expanding crack with a uniform stress drop. The former is peaked at the center, the latter at the tips (as smoothed by the slip-weakening regions); each varies by only a factor of 2 over the central $86 \%$ of the crack. To the extent that a factor approaching two appears small on a logarithmic scale, then the velocity and stress drop may appear quasiuniform over a wide range of the ratios of the coefficients $(\dot{\Delta \tau}) L$ and $\Delta \tau \dot{L}$. Variations in this ratio determine the extent to which the velocity is peaked at the crack center or near the tips, as in Figure 6 and others to follow.

\subsection{From Localized Acceleration to Expansion}

[36] To gain some insight into the transition to expansion of the nucleation zone, we can substitute the full evolution equation $\dot{\theta}=1-\Omega$ into equation (9) to obtain

$$
-a \sigma \frac{\dot{V}}{V^{2}}-\frac{b \sigma}{V \theta}+\frac{b \sigma}{D_{c}}=-\frac{\dot{\tau}}{V}=W^{*} .
$$


Here $W^{*}$ may be interpreted as an "effective" or bulk slipweakening rate, with contributions from the direct effect $\left(a \sigma \dot{V} / V^{2}\right.$; essentially a nonlinear viscosity), fault healing $(b \sigma / V \theta)$, and slip weakening $\left(b \sigma / D_{c}\right)$. In the no-healing limit the $b \sigma / V \theta$ term is negligible, and using the separable formulation $V=V_{o}(t) \mathcal{V}(\xi)$ (equation (11)) we have

$$
-\frac{a \sigma}{\mathcal{V}(\xi)} \frac{\dot{V}_{o}}{V_{o}^{2}}+\frac{b \sigma}{D_{c}}=W^{*}
$$

Substituting the $K=0$ fixed length result $\dot{V}_{o} / V_{o}^{2}=$ $0.3781\left(b / a D_{c}\right),(30)$ indicates a bulk weakening rate of $0.6219\left(b \sigma / D_{c}\right)$ at the crack center $(\mathcal{V}=1)$. Near the crack tips, where $\mathcal{V}$ approaches zero, the $b \sigma / D_{c}$ term is negligible and the bulk "weakening" rate is negative. This is required to satisfy the $K=0$ criterion for a stationary crack. In essence, near the crack center the evolution effect dominates and the fault weakens during the approach to instability, while near the ends the direct effect dominates and the fault strengthens (as can be seen, for example, by dividing equation $(23)$ by $V(\xi, t) / V(\xi, 0))$.

[37] Once $\Omega$ approaches unity near the crack center, the healing term is no longer negligible and the bulk weakening rate decreases. In the limit of zero weakening, elasticity requires that further slip at the crack center be accompanied by an increase in crack length. However, this is readily achieved, since the stresses near the crack tips are very large. Zero acceleration at the crack center, coupled with continued large accelerations at the tips, ensures that crack expansion occurs simultaneously with slip at the center. One might expect qualitatively similar results for a reduced but finite weakening rate at the crack center, and an analogy with nonlinear slip-weakening behavior indicates that this is the case. If the slip-weakening curve has an inflection point, such that the largest weakening rate occurs at a finite slip distance, then localization will progress for as long as the weakening rate is increasing, but expansion ensues after some portion of the nucleation zone passes through the inflection [Suo et al., 1992; Ampuero, 2002]. The minimum nucleation length in this case (analogous to our $L_{\min }$ ) is sensitive to the maximum slope of the friction law, a result that follows from dimensional analysis. The transition from localization to expansion becomes sharper as the difference in weakening rates across the inflection becomes more pronounced. The main qualitative difference between the slip-weakening and rate-and-state cases lies in how slow (quasi-static) motion is enforced. In the slip-weakening case stability requires a reduction in driving stress with slip, while in rate-and-state friction the nonlinear viscosity introduced by the direct effect allows for quasi-static acceleration of slip under constant load.

\subsection{Nucleation Length}

[38] We start from the observation of a near-constant $\Omega$ in the interior of the nucleation zone, and note that this implies $\dot{V} / V+\dot{\theta} / \theta=0$. With $\dot{\theta}=1-\Omega$, this leads to

$$
\frac{\dot{V}}{V^{2}}=\frac{1-\Omega^{-1}}{D_{c}} \equiv \frac{C_{1}}{D_{c}}
$$

As this is identical in form to equation (15), we have

$$
V=V(0)\left(1-t / t^{*}\right)^{-1} ; t^{*} \equiv C_{1}^{-1} D_{c} / V(0)
$$

That is, the slip velocity again increases as the inverse time to instability, but with $C_{1}$ now given by (31). This result is confirmed by the numerical simulations in that plots of $\log (V)$ versus $\log \left(t^{*}-t\right)$ for all the simulations with the same $a / b$ (meaning nearly the same $\Omega$ ) are indistinguishable over the time window that $\Omega$ is $\sim$ constant, and have the slope and intercept predicted by (32). As the no-healing approximation was used by Dieterich [1994] to derive Omori-law-type aftershock statistics from rate and state friction, the fact that the constant-weakening limit shares this property suggests that similar behavior might be found in this regime as well, although more work is certainly necessary to determine if this is so.

[39] One must also ask what aspect of the underlying mechanics gives rise to the near-constant $\Omega$. Substituting $\Omega$ for $V \theta / D_{c}$ in equation (1) yields

$$
\frac{\tau}{\sigma}=f^{*}+a \ln \frac{V}{V^{*}}+b\left[\ln (\Omega)-\ln \frac{V}{V^{*}}\right] .
$$

This is offset from the steady state curve by $b \ln (\Omega)$, so to the extent that $\Omega$ is constant differentiating with respect to time gives the same result as for steady state sliding:

$$
\frac{\dot{\tau}}{\sigma}=(a-b) \frac{\dot{V}}{V}
$$

From equilibrium, once nucleation is well underway and $\dot{\tau}^{\infty}$ is negligible, the effective elastic stiffness $k^{*}$ may be determined by dividing equation (34) by $V$ and using (31):

$$
k^{*}=-\frac{\dot{\tau}^{e l}}{V} \approx-\frac{\dot{\tau}}{V}=\frac{\sigma(b-a)}{D_{c}}\left(1-\Omega^{-1}\right) .
$$

For a given set of initial conditions embodied by $\Omega,(35)$ gives the unique value of the stiffness required to maintain $\Omega$ constant or, equivalently, cause the trajectory on a plot of $\tau$ versus $V$ to parallel the steady state line. The same expression, albeit in a somewhat different form, was obtained previously by Ranjith and Rice [2003] for a constant-stiffness spring-block-slider with zero load point velocity. A smaller stiffness for the given $\Omega$ causes $\Omega$ and $V$ to increase monotonically until instability, whereas a larger stiffness leads to a monotonically decreasing $\Omega$ and a velocity that ultimately tends to zero. We find in the numerical simulations that $-\dot{\tau} / V$ (i.e., $k^{*}$ ) tends to oscillate around the value given by (35) (evaluated using the current $\Omega$ ), a point we return to at the end of this section. First we examine what determines the particular $\left(k^{*}, \Omega\right)$ pair the simulations evolve to. This is tied to the size of the nucleation zone, which we estimate next.

[40] As with elastodynamic ruptures on a rate-and-state fault, the peak stress near the crack tip can be estimated quite well by assuming the slip rate to jump instantaneously to the value $V$ at the crack center while $\theta$ remains constant 


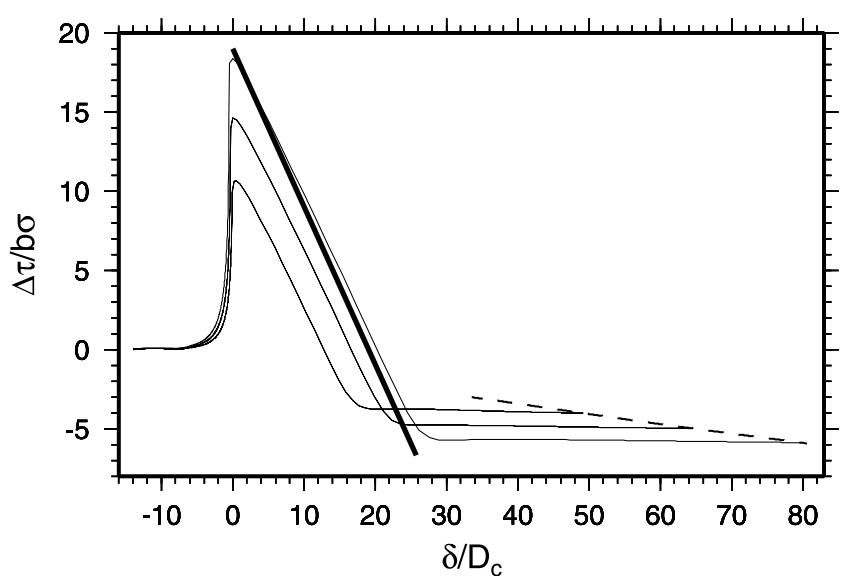

Figure 7. Normalized shear stress change (thin lines), $\Delta \tau / b \sigma$, as a function of normalized slip, $\delta / D_{c}$, for three snapshots during the crack-like expansion of a nucleation zone for $a / b=0.8$ (as in Figure 6 but for a localized loading rate on an initially steady state surface; solid circles in Figures 2 and 3). The indicated slip is that which has accumulated since the beginning of the simulation minus that at the "crack tip" (the location of the peak stress) in the final snapshot (the curve with the largest peak stress). The right endpoints of the curves correspond to the center of the nucleation zone. The bold line indicates a slip-weakening rate of $b \sigma / D_{c}$, drawn with the peak-to-residual and ambient-toresidual stress drops given by (36) and (40), evaluated using $V$ and $\Omega$ (at the crack center) and $\theta_{i}$ (at the crack tip) extracted from the final snapshot, and shows good agreement with the numerical simulation. The dashed line shows the bulk slip weakening rate $W^{*}$ at the center of the nucleation zone over the time window that $\Omega$ is quasi-constant.

(Figure 7). Subtracting (33) from (1) and simplifying, the peak-to-residual stress drop $\Delta \tau^{p-r}$ is

$$
\Delta \tau^{p-r}=\sigma b \ln \left(\frac{V \theta_{i}}{D_{c} \Omega}\right)
$$

where $\theta_{i}$ denotes the value of $\theta$ just prior to the arrival of the tip. Given the abrupt jump in velocity, near the tip $\Omega \gg 1$ (e.g., Figure 1f). Assuming $V$ is maintained constant, as $\theta$ evolves the effective slip-weakening rate $W^{*}$ (from equation (29) with $\dot{V}=0$ ) is $b \sigma / D_{c}$, at least until near-steady state conditions are reached. The effective slip-weakening distance $\delta_{c}$ is then

$$
\delta_{c}=\frac{\Delta \tau^{p-r}}{W^{*}}=D_{c} \ln \left(\frac{V \theta_{i}}{D_{c} \Omega}\right) .
$$

For $\Omega=1$ this reduces to the result of Bizzarri and Cocco [2003] that the effective slip-weakening distance is larger than $D_{c}$ by the logarithm of the ratio of $\theta_{i}$ to the steady state $\theta$ at seismic slip speeds (see also Dieterich and Kilgore [1996]).

[41] For a constant slip-weakening rate the fracture energy $G_{c}$ is $0.5 \Delta \tau^{p-r} \delta_{c}$, so combining (36) and (37) yields

$$
G_{c}=\frac{\sigma b D_{c}}{2}\left[\ln \left(\frac{V \theta_{i}}{D_{c} \Omega}\right)\right]^{2}
$$

For an equilibrium crack $G_{c}$ is balanced by the reduction in mechanical energy per increment of crack length, given by

$$
G=\frac{\pi}{2} \frac{L}{\mu^{\prime}} \Delta \tau^{2},
$$

where $\Delta \tau$ is the ambient-to-residual stress drop [Lawn, 1993]. To estimate $\Delta \tau$, we can write the initial stress as equivalent to that due to slip at a hypothetical (not necessarily realized) steady state background velocity $V_{b g}$. Substituting this into (5) and subtracting (33),

$$
G=\frac{\pi}{2} \frac{L}{\mu^{\prime}}\left[-\sigma b \ln \Omega+\sigma(b-a) \ln \frac{V}{V_{b g}}\right]^{2}
$$

Equating $G$ with $G_{c}$, the equilibrium crack length $L_{c}$ is found to be

$$
L_{c}=\frac{1}{\pi}\left(\frac{b}{b-a}\right)^{2} L_{b}\left[\frac{\ln \frac{V}{D_{c} / \theta_{i}}-\ln \Omega}{\ln \frac{V}{V_{b g}}-\frac{b}{b-a} \ln \Omega}\right]^{2}
$$

For $\Omega$ a constant near 1 , the bracketed expression in (41) approaches 1 in the limit $V \gg V_{b g}, D_{c} / \theta_{i}$. In all our simulations $D_{c} / \theta_{i}>V_{b g}$ because prior to the arrival of the crack tip $\theta_{i}$ had been reduced from the background value (either because the nucleation zone had been larger or as a result of the approaching stress concentration); this seems likely to be a rather general result. Thus the bracketed expression asymptotically approaches 1 from below, which explains the slow (and slowing) expansion of the nucleation zones in Figure 6. Writing $L_{\infty}$ for this limiting value of $L_{c}$,

$$
L_{\infty} \approx \frac{1}{\pi}\left(\frac{b}{b-a}\right)^{2} L_{b}
$$

The dependence on $[b /(b-a)]^{2}$ implies that the nucleation length can grow to be much larger than $L_{\nu}$ as $a$ approaches $b$ (e.g., by factors of 10 and 100 for $a / b=0.85$ and 0.95 , respectively).

[42] Because the crack has a near-constant stress drop and is growing only slowly, the effective stiffness is a quasi-constant $\sim \mu^{\prime} / 2 L_{\infty}$. Setting this equal to the right side of (35) and using the definition of $C_{1}$ from (31) we obtain

$$
C_{1} \equiv 1-\Omega^{-1} \approx \frac{\pi}{2} \frac{b-a}{b}
$$

implying that the interior of the nucleation zone approaches steady state $(\Omega=1)$ more and more closely as $a$ approaches $b$. Combining this result with (32), the time remaining to instability $t^{*}-t$ is

$$
t^{*}-t=\frac{2}{\pi} \frac{b}{b-a} \frac{D_{c}}{V(t)},
$$




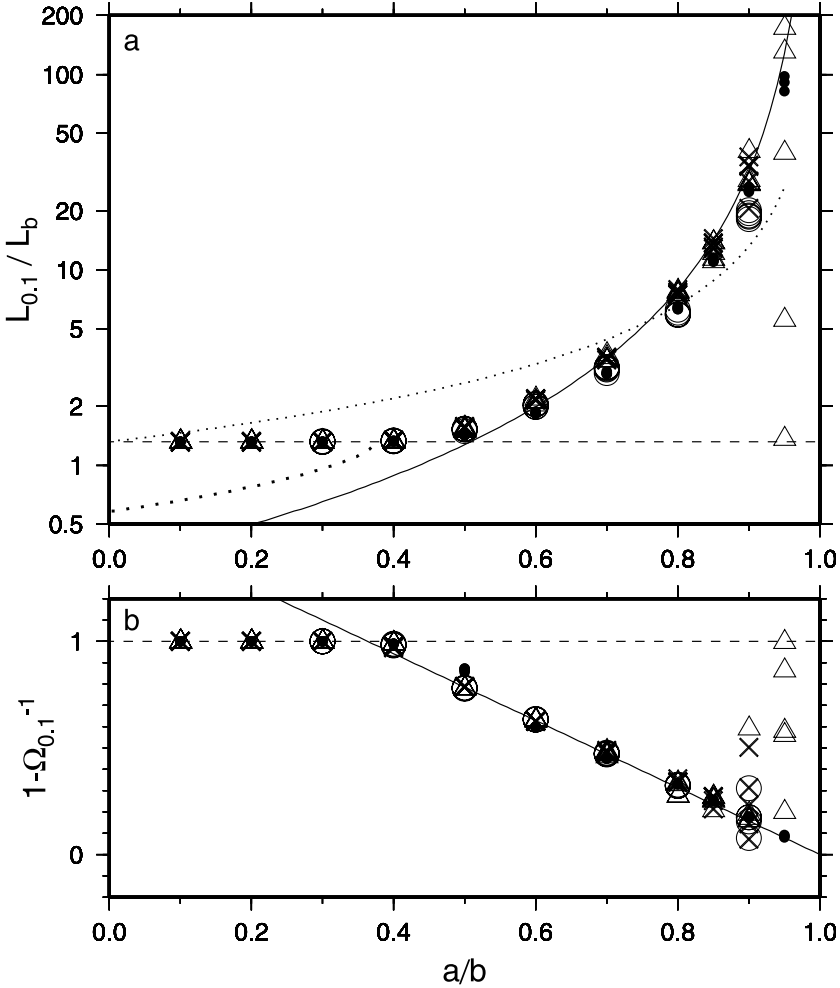

Figure 8. (a) Dimensionless nucleation length and (b) $1-$ $\Omega^{-1}$ at the center of the nucleation zone, evaluated when the maximum slip speed reaches $0.1 \mathrm{~m} / \mathrm{s}$. Solid circles, open circles, and triangles represent the same loading conditions as in Figure 2; crosses have the same boundary conditions as the solid circles but an initial velocity of $10^{-11}$ rather than $10^{-9} \mathrm{~m} / \mathrm{s}$. Unlike Figure 2, for the open circles each of the five values of $b$ at the same $a / b$ used a different realization of the random initial conditions (the same realizations led to values of $L_{0.1}$ and $\Omega_{0.1}$ that were nearly indistinguishable). The horizontal dashed lines represent the $K=0$ fixed length solution. The solid lines represent the constant-weakening asymptotic estimates (equations (42) and (43)). In Figure 8a, the bottom dotted line indicates the minimum nucleation length for $a / b<0.3781$, and the top dotted line shows scaling as $(b-a)^{-1}$, as in Figure 2.

where $V(t)$ is the current slip speed in the interior of the nucleation zone. The corresponding result for the no-healing limit is (combining (22) and (16))

$$
t^{*}-t=2.645 \frac{a}{b} \frac{D_{c}}{V(t)} .
$$

Equations (44) and (45) show that in the no-healing limit the time to instability is of order $D_{c} / V(t)$ for $a / b \sim 1$, but that in the constant-weakening limit this time becomes unbounded (the two estimates are nearly identical for $a / b=$ 0.5 , as are the respective estimates of nucleation length).

[43] In a practical sense, we are interested in the size of the nucleation zone as it begins to radiate seismic energy. We define $L_{0.1}$ to be the nucleation length at the time that the maximum slip speed reaches $0.1 \mathrm{~m} / \mathrm{s}$, as this is roughly the velocity at which elastodynamics becomes important
(Appendix A2). Figure 8 shows that the $L_{\infty}$ defined in (42) provides a good approximation to the values of $L_{0.1}$ determined from the simulations. The discrepancy of some tens of percent in the case of the heterogeneous initial conditions (open circles) is attributable at least in part to the limiting length not yet being reached. The bottom panel in Figure 8 compares the value of $1-\Omega^{-1}$ at the center of the nucleation zone to the prediction from (43), again at a velocity of $10^{-1} \mathrm{~m} / \mathrm{s}$. In this case the extent of the agreement is perhaps surprising, at least for $a / b \lesssim 0.8$, in that (43) assumes that $L_{\infty}$ has already been reached. This is not the case, for example, with the open circles for $a / b=0.7$ and 0.8. In fact, the agreement is such as to lead us to suspect that there may be a more direct route to the same result (note also the good fit at $a / b=0.5$, despite the suggestion from the top panel that equation (42) may not be applicable at this $a / b$ ).

[44] Further insight into the crack expansion phase of nucleation can be obtained by comparing snapshots of the velocity distribution to the evolution of $\Omega(0, t)$ and $k^{*}(0, t)$, particularly when the latter are somewhat variable. In general this variability increases as $a$ approaches $b$; Figure 9 shows examples for $a / b=0.9$. The long and short dark bars in the left panels indicate $L_{\infty}$ and $L_{\nu}$. The top panels are for the peaked initial stress (triangles in Figures 2 and 8). After an initial expansion out to $L>L_{\infty}$ (associated with $\Omega<1$ and decreasing velocities at the origin), the nucleation zone localizes to a region substantially smaller than $L_{\infty}$ as $\Omega$ first exceeds 2 and then decreases to near steady state (for $V(0, t)$ between $\sim 10^{-10}$ and $10^{-7} \mathrm{~m} / \mathrm{s}$ ). The nucleation zone then expands again at a quasi-constant $\Omega$, reaching nearly the predicted $L_{\infty}$ by the time $V(0, t)$ reaches $\sim 10^{-4} \mathrm{~m} / \mathrm{s}$. Thereafter, the nucleation zone accelerates to well beyond elastodynamic slip speeds while maintaining essentially the same length. At still larger velocities the excursions of $\Omega$ and $k^{*}$ become greater, and the nucleation zone alternates between periods of localization for large $\Omega$ and expansion for small $\Omega$. Although the simulations are of course invalid at such large speeds, we show them because they illustrate the behavior the underlying quasi-static equations; stated differently, different loading conditions could plausibly lead to similarly shaped velocity profiles with absolute velocities orders of magnitude less.

[45] For the heterogeneous initial conditions (bottom panels; open circles in Figures 2 and 8), the nucleation zone is only $2 / 3$ of $L_{\infty}$ but still growing when $V(0, t)$ reaches $0.1 \mathrm{~m} / \mathrm{s}$. The tendency of $k^{*}$ to oscillate around the value $k_{\Omega}$ needed to maintain the expected $\Omega$ is more obvious in this case, particularly at less than elastodynamic speeds, as is the phase lag between $k^{*}$ and $\Omega$ (the latter decreasing when the former exceeds that given by equation (35)). For this example the variability of $\Omega$ and $k^{*}$ would likely be reduced if they were defined as an average over the interior of the nucleation zone, rather than as a point measurement.

[46] The negative feedback that maintains a quasiconstant $\Omega$ seems to work in the following way: A low $\Omega$ (increased healing) is associated with a reduced acceleration at the crack center relative to the edges, and a velocity profile that is concave-upward or at least flatter than the elliptical distribution associated with a constant stress drop (for example, the local minima in $k^{*}$ for $V(0, t)$ near $10^{2}$ and 

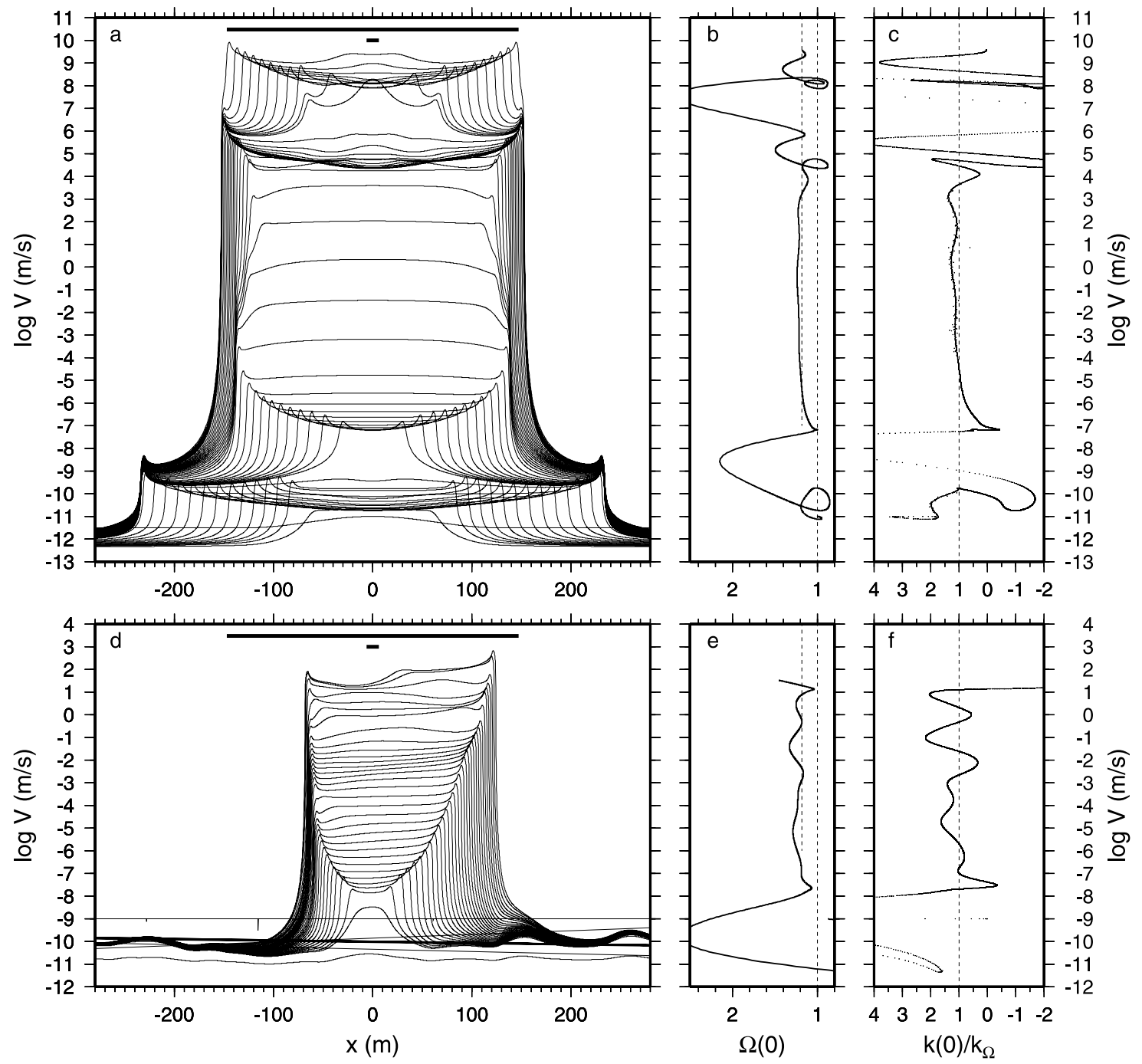

Figure 9. Simulation results for $a / b=0.9$. $(\mathrm{a}-\mathrm{c})$ Locally peaked load (triangles in Figures 2 and 8$)$; (df) randomly heterogeneous state (open circles in Figures 2 and 8). Figures 9a and 9d show snapshots of the velocity; the top and bottom horizontal bars represent $L_{\infty}$ and $L_{\nu}$, respectively. Figures $9 \mathrm{~b}$ and $9 \mathrm{e}$ show the evolution of $\Omega$ evaluated at $x=0$; the right dashed line indicates steady state, and the left dashed line indicates the prediction from equation (43). Figures $9 \mathrm{c}$ and $9 \mathrm{f}$ show the effective stiffness $k^{*}=\dot{\tau} / V$, evaluated at $x=0$ and normalized by the value $k_{\Omega}$ required from equation (35) to maintain $\Omega$ at the value indicated by equation (43). There is an imperfect feedback mechanism that tends to maintain $\Omega$ near the predicted value; excursions to $\Omega \gtrsim 3$ are associated with pronounced localization.

$10^{4} \mathrm{~m} / \mathrm{s}$ in Figures $\left.9 \mathrm{a}-9 \mathrm{c}\right)$. This reduces the effective stiffness (the crack edges load the center), which, following Ranjith and Rice [2003] leads to an increasing $\Omega$. Conversely, if $\Omega$ becomes too large for the current stiffness, healing becomes less important and the center of the nucleation zone accelerates more than in the constant $\Omega$ case. If this acceleration leaves the neighboring regions behind, the effective stiffness increases, which in turn decreases $\Omega$ (for example, the local maxima in $k^{*}$ for $V(0, t)$ near $10^{\circ}$ and $10^{3} \mathrm{~m} / \mathrm{s}$ in the top row).

[47] In general we find increasing complexity in the velocity profiles during nucleation, and increasingly large excursions of $\Omega$ from the expected value, with increasing $a / b$. Plausibly this is because the time to instability in the constant-weakening regime becomes unbounded as $a / b$ approaches 1 (equation (44)), allowing some other mode of slip to dominate. For $a / b=0.9$, and to a greater extent for $a / b=0.95$ (Figure 10), this complexity begins at slip speeds less than $0.1 \mathrm{~m} / \mathrm{s}$. Such behavior is responsible for the increasing scatter of $1-\Omega^{-1}$ for $a / b>0.9$ in Figure 8. In Figure 10, deceleration of the center of the nucleation zone is sometimes followed by excursions of $\Omega$ to such large values that over a substantial velocity range the nucleation length approaches $L_{\nu}$. This is the cause of the large scatter in $L_{0.1}$ for the initially peaked loads (triangles) with $a / b=0.95$ in Figure 8.

\subsection{Speculations Concerning Seismic Nucleation Phases}

[48] The large nucleation length for $a / b$ approaching 1 warrants some comments concerning the possible detection of seismic nucleation phases. Ellsworth and Beroza [1995, 

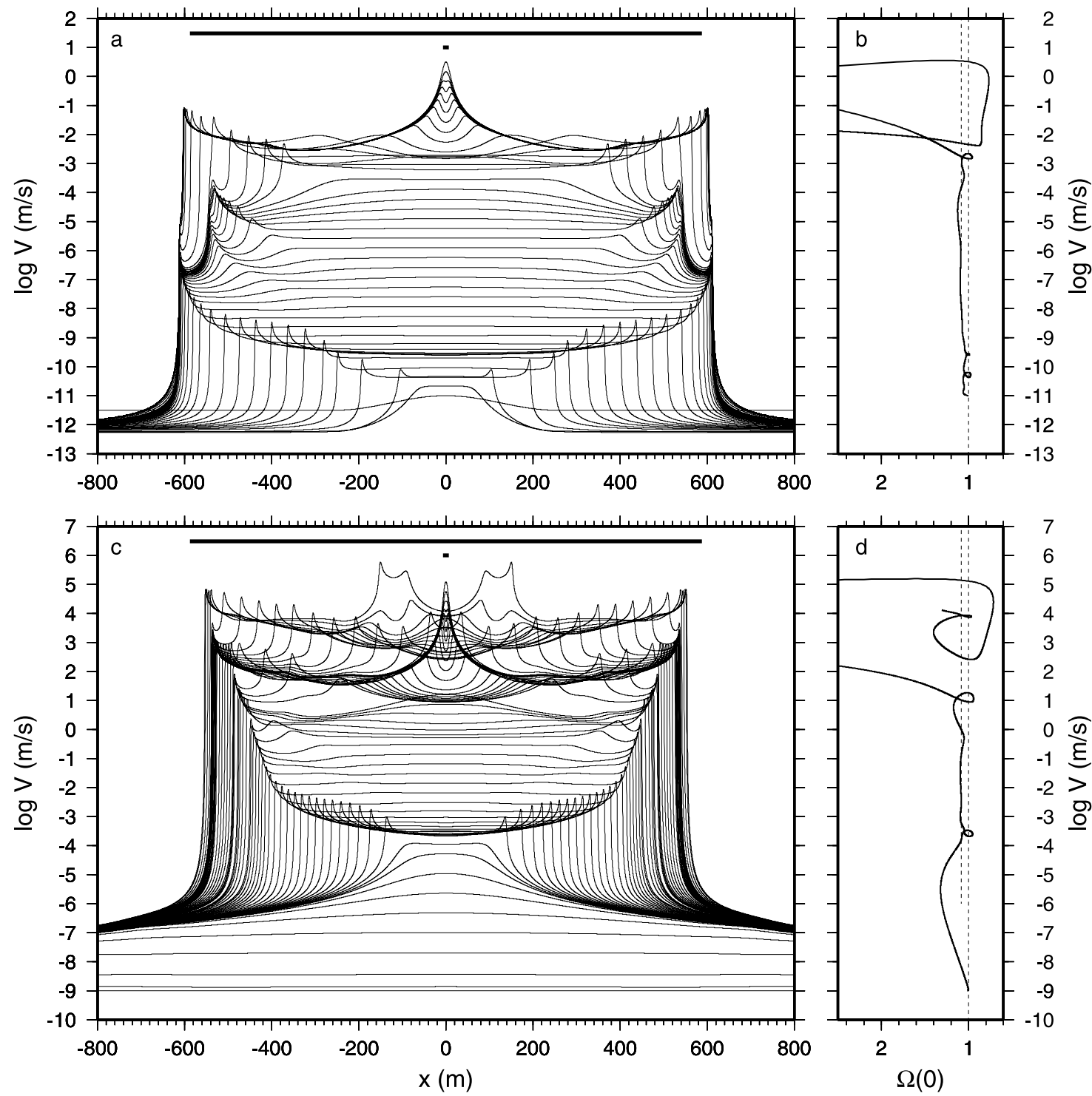

Figure 10. Simulation results for $a / b=0.95$. (a and b) Locally peaked load (triangles in Figures 2 and 8); (c and d) localized loading rate (solid circles in Figures 2 and 8). Horizontal bars represent $L_{\infty}$ and $L_{\nu}$ as in Figure 9.

1998] find that many earthquakes are preceded by a short $(<1$ s) period during which the moment rate increases significantly less rapidly than expected for self-similar expansion of a dynamic crack. They identify the subsequent portion of the seismogram, that at low gain might be identified as the start of the rupture, as the "breakaway phase" marking the transition from nucleation to dynamic crack expansion. While this might be the expected signature of a gradual transition from aseismic to seismic slip, they also observe that the nucleation phase typically includes periods of decreasing moment acceleration.

[49] To address at least qualitatively the possible seismic signature of nucleation, we add to the simulations of Figures $9 \mathrm{a}$ and $10 \mathrm{a}$ the local radiation damping term $\dot{\tau}^{\mathrm{rad}}$ $=\dot{V} \mu / 2 c_{s}$, where $\mu$ is the shear modulus and $c_{s}$ is the shear wave speed (Appendix A2). The resulting velocity snapshots and moment rate and acceleration (the latter two computed assuming radial symmetry, as done by Lapusta and Rice [2003]) are shown in Figure 11. The moment acceleration for $a / b=0.9$ in Figure 11c shows that far from being gradual, the transition from nucleation to propagation is punctuated by a small "bang" marking the last (most rapidly accelerating) stage of nucleation prior to the dominance of radiation damping (at $t=0 \mathrm{~s}$ ). Radiation damping then drops the acceleration of the rapidly slipping region to near zero; the subsequent slow increase in moment acceleration is due to the breakaway phase. Fully elastodynamic simulations show that the peak moment acceleration at $t=0 \mathrm{~s}$ is somewhat larger than in the radiation damping case, but that rupture growth is more rapid so that the subsequent drop in moment acceleration is not as dramatic (the local minimum is $\sim 70 \%$ of the prior peak value, rather than near zero).

[50] Figure 11d is similar except that the excursions of the nucleation zone between the disparate length scales $L_{\infty}$ and $L_{\nu}$ produce a larger and more complex moment acceleration. 

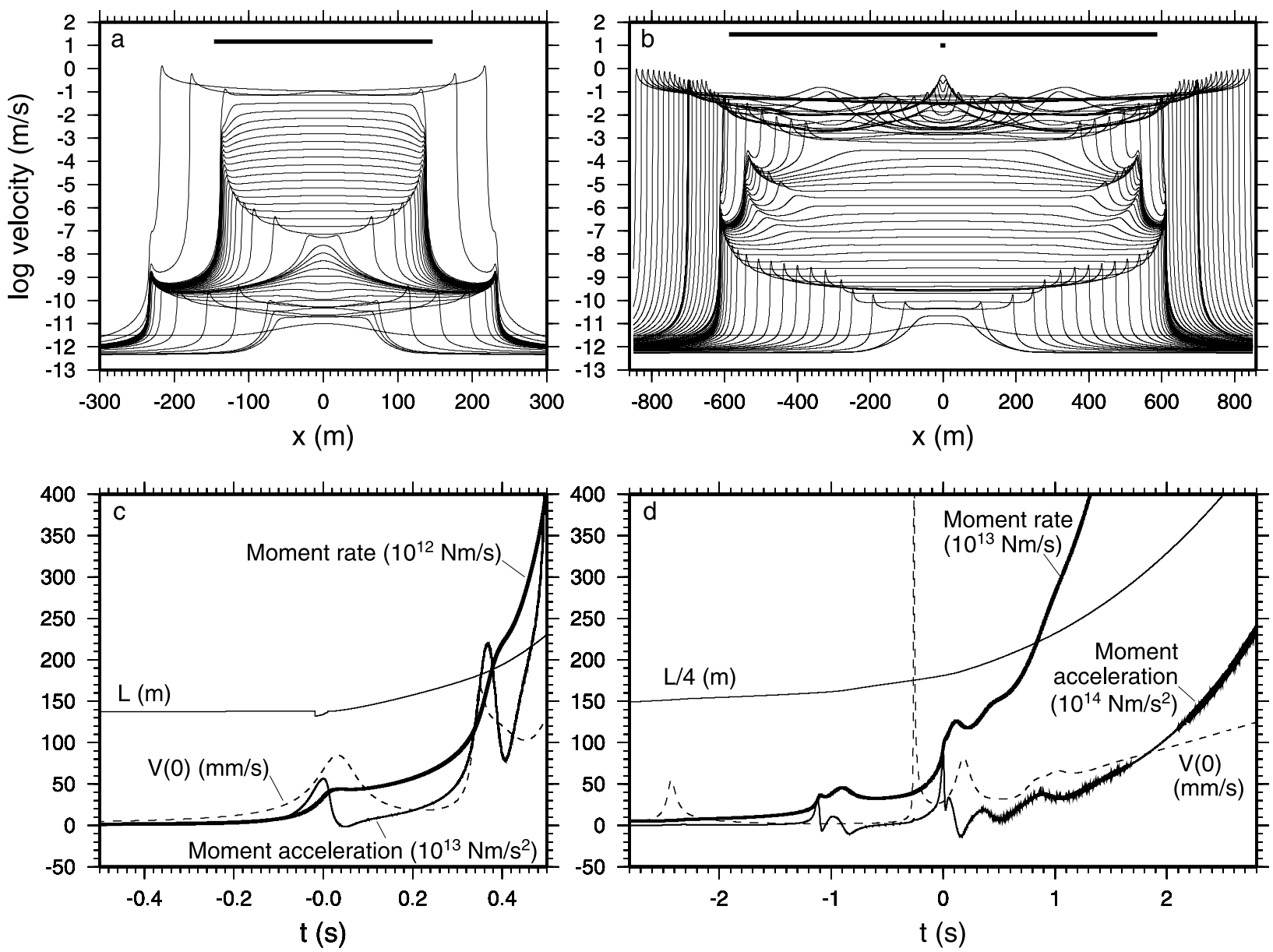

Figure 11. ( $a$ and b) Results for the same simulations as in Figures 9a and 10a but with the inclusion of radiation damping. (c and d) Velocity at the origin $V(0)$, moment rate (computed assuming radial symmetry), moment acceleration, and crack length $L$ as functions of time. Zero time is arbitrarily set to be at the sharp peak in moment acceleration marking the transition from nucleation to propagation. Excursions to large $V(0)$ at -2.4 and $-0.25 \mathrm{~s}$ in Figure $11 \mathrm{~d}$ do not contribute significantly to the moment rate because of the small area implied.

Lapusta and Rice [2003] showed that an initially irregular moment rate could arise when the rupture front encounters stress heterogeneities left over from previous earthquakes outside the nucleation zone. Our simulation adds to that the possibility that this complexity can be due to heterogeneous slip within the nucleation zone itself, at least for $a / b$ near 1 , and furthermore that such slip can arise from quite smooth initial stresses as a result of the nonlinearity of the governing equations.

[51] To assess the detectability of the nucleation phase, we can compare the computed moment acceleration (to which far-field velocity seismograms are proportional) to that expected for a "standard" (growing) circular rupture of radius $l$. For a constant stress drop $\Delta \tau$ and rupture velocity $V_{r}$,

$$
\begin{gathered}
M_{o}=\mu \pi l^{2} \delta=\alpha_{1} \Delta \tau l^{3}, \\
\ddot{M}_{o}=6 \alpha_{1} \Delta \tau V_{r}^{2} l,
\end{gathered}
$$

where the constant $\alpha_{1}=16 / 7$ for static elasticity but is somewhat smaller at large rupture speeds. For $\Delta \tau=3 \mathrm{MPa}$ and $V_{r}=2600 \mathrm{~m} / \mathrm{s}$, we find $\ddot{M}_{o} \sim 5 \times 10^{15} \mathrm{Nm} / \mathrm{s}^{2}$ for $l=$ $20 \mathrm{~m}$ (corresponding to a magnitude 1 earthquake, using the moment-magnitude relation $\log \left[M_{o} / \mathrm{N} \mathrm{m}\right]=1.0 M+9.8$ of Abercrombie [1996]). This is roughly the size of the event at $t=-1 \mathrm{~s}$ in Figure $11 \mathrm{~d}$. The event at $t=0 \mathrm{~s}$, with $\ddot{M}_{o}=$ $10^{16} \mathrm{Nm} / \mathrm{s}^{2}$, would be comparable to a "standard" earthquake with $l=40 \mathrm{~m}$ (magnitude $\sim 2$ ).

[52] To estimate how the moment acceleration during nucleation varies with the governing parameters, we assume that nucleation proceeds smoothly over the length scale $L_{\infty}$ until the onset of radiation damping. In this case the moment rate and acceleration are

$$
\dot{M}_{\nu}=\mu \pi L_{\infty}^{2} V ; \quad \ddot{M}_{\nu}=\mu \pi L_{\infty}^{2} \dot{V} .
$$

Substituting (42) for $L_{\infty}$ and using (31) and (43) to evaluate $\dot{V}$, the estimated moment acceleration at $V=V_{\mathrm{dyn}}=2(a-$ $b) \sigma c_{s} / \mu$ (Appendix A2) is

$$
\ddot{M}_{\nu} \approx \mu D_{c}\left(\frac{b}{b-a}\right) c_{s}^{2} .
$$


Equation (49) accurately predicts the moment acceleration at the end of nucleation in Figure 11c (at $t=0$ ), but underestimates that in Figure $11 \mathrm{~d}$ by nearly an order of magnitude, apparently because of the complexity associated with the cycling between the length scales $L_{\infty}$ and $L_{\nu}$. Although the value of $D_{c}$ used here $(400 \mu \mathrm{m})$ is large by laboratory standards, equation (49) shows that similar moment accelerations might be expected for $D_{c}=130 \mu \mathrm{m}$ (near the upper end of the lab range) if $\mu$ were increased from 8.67 to $26 \mathrm{GPa}$ (we used a low modulus to account for the possibly fractured nature of the fault zone). Thus we conclude that nucleation under the aging law might produce detectable seismic signals for large but plausible values of $a / b(\gtrsim 0.95)$ and $D_{c}(\gtrsim 100 \mu \mathrm{m})$, with the caveat that additional fully elastodynamic simulations are needed to verify this.

\subsection{Why So Big?}

[53] The aging law promotes such large nucleation lengths as $a / b$ approaches 1 as a direct result of the large fracture energy that it implies. For both the aging and the slip laws, following a step increase in slip speed the peakto-residual stress drop increases as $\ln \left(V / V_{b g}\right)$ (equation (36)), but only for the aging law does the effective slipweakening distance also increase with slip speed (again as $\ln \left[V / V_{b g}\right]$; equation (37)). As the fracture energy is the product of these quantities, integrating (8) shows that the effective fracture energy is only $\sim\left(\sigma b D_{c}\right) \ln \left(V / V_{b g}\right)$ for the slip law, compared to $\sim\left(\sigma b D_{c} / 2\right)\left(\left[\ln \left(V / V_{b g}\right)\right]^{2}\right.$ (equation (38)) for the aging law. This extra order of $\ln \left(V / V_{b g}\right)$ in turn requires a large equilibrium crack, given the available stress drop within the interior of the nucleation zone.

[54] In fact, the prediction of the aging law that the effective slip weakening distance increases with velocity has not been verified in the laboratory [Nakatani, 2001], and seems to be at odds with the intuitive notion that the slip distance required to renew the population of contacts should be largely independent of that velocity. Nonetheless, it must be borne in mind that nearly all velocity-stepping experiments to date have been limited to changes of a single order of magnitude. Our analysis demonstrates that in the constant-weakening regime the nucleation length is determined by the response of the sliding surface to sudden velocity increases of many orders of magnitude.

\section{Onset of Nucleation}

[55] We have shown that there are two regimes of nucleation distinguished by the behavior of $\Omega$ in the interior: Fixed length growth for $\Omega \gg 1$, and crack-like expansion for $\Omega \sim 1$. For the loading conditions we have considered, these regimes are separated by a value of $a / b$ that is low by laboratory standards. However, Figure 2 shows that the loading conditions also play a role, and could potentially place nucleation in the regime $\Omega \gg 1$ even for large $a / b$. For example, a region subjected to a stress step $\Delta \tau$ undergoes an instantaneous increase in slip speed (and hence $\Omega$ ) of exp $[\Delta \tau / a \sigma]$ (equation (1)), which could plausibly reach several orders of magnitude locally (although equations (22) and (23) show that such large values may not persist over a large range of velocities, once the fixed length solution is approached, and equation (45) for the time to instability in turn shows that only the very earliest aftershocks might remain in the no-healing limit all the way to dynamic slip speeds). Here we neglect such early aftershocks and focus on slow loading conditions akin to those of our simulations.

[56] At some point in all simulations that reach instability, the nucleation zone passes through steady state with $\Omega$ increasing. For $a / b<0.3781$ this increase persists until instability, while for larger values of $a / b$ it ultimately decreases. The maximum value of $\Omega$ attained in the latter case determines whether and over what range of velocities the no-healing fixed length solution is followed. Dieterich [1992] suggested that $\Omega$ commonly greatly exceeds 1 as instability is approached, but our numerical results (e.g., Figure 3) show that for slow loading this is generally not the case as $a$ approaches $b$. In this section we examine the evolution of $\Omega$ during the earliest phases of nucleation, prior to the onset of either the no-healing or constant-weakening regimes. Because this evolution is closely tied to the effective elastic stiffness of the nucleation zone, we begin by discussing the related question of whether the nucleation zone expands or contracts.

\subsection{Expansion or Localization?}

[57] A glance at Figures 9 and 10 reveals numerous instances of expansion and contraction of the nucleation zone. Some, such as the initial expansions out to $L_{\infty}$, can be understood in terms of the fracture energy balance of section 4. Others, such as the occasional localizations toward $L_{\nu}$ at large slip speeds, can be rationalized as a byproduct of excursions to moderate values of $\Omega$ and an approach to the no-healing fixed length solution. Similarly, the initial expansion out to $L>L_{\infty}$ in Figure $9 \mathrm{a}$ is associated with $\Omega<1$, whereas otherwise identical simulations with initial conditions of (for example) $\Omega=2$ over a distance $L_{\infty} /$ 2 initially undergo localization. Clearly, larger values of $\Omega$ help promote localization. However, as we discuss below and in more detail in Appendix B, this is not the full story because even in the no-healing limit both expansion and localization are possible.

[58] Figure 12 shows the evolution of $V$ and $\Omega$ for a fault that is initially at steady state with $V=10^{-9} \mathrm{~m} / \mathrm{s}$, and for which $\dot{\tau}^{\infty}=0$ except over $|x| \leq 0.1 L_{b-a}$ (solid circles in Figures 2 and 8). The upper panels show an initial expansion of the nucleation zone out to distances $L \gg L_{\infty}$, followed by localization. For more heterogeneous initial conditions we do not observe a well-defined expansion to large $L$, presumably because the (small) elastic stressing rate due to this phase would be overwhelmed by the stressing rate that arises from the initial heterogeneity (i.e., multiple peaks in slip velocity). However, all our simulations undergo some localization prior to reaching a minimum nucleation length (indeed, this is how the $L_{\text {min }}$ Figure 2 were defined). Localization from longer-wavelength initial perturbations was also noted by Dieterich [1992].

[59] It is useful to view the question of expansion or contraction of the nucleation zone as a competition between elasticity, which acts to smooth lateral variations in velocity and thus enlarge the nucleation zone, and slip weakening, which promotes localization. That the initial expansion in Figure 12 is due to elasticity is self-evident: Given that the nonzero remote stressing rate is restricted to a region smaller than $L_{\nu}$, only the stress increase for $x \gtrsim L$ due to slip over $x \lesssim L$ can cause $L$ to increase. This initial 

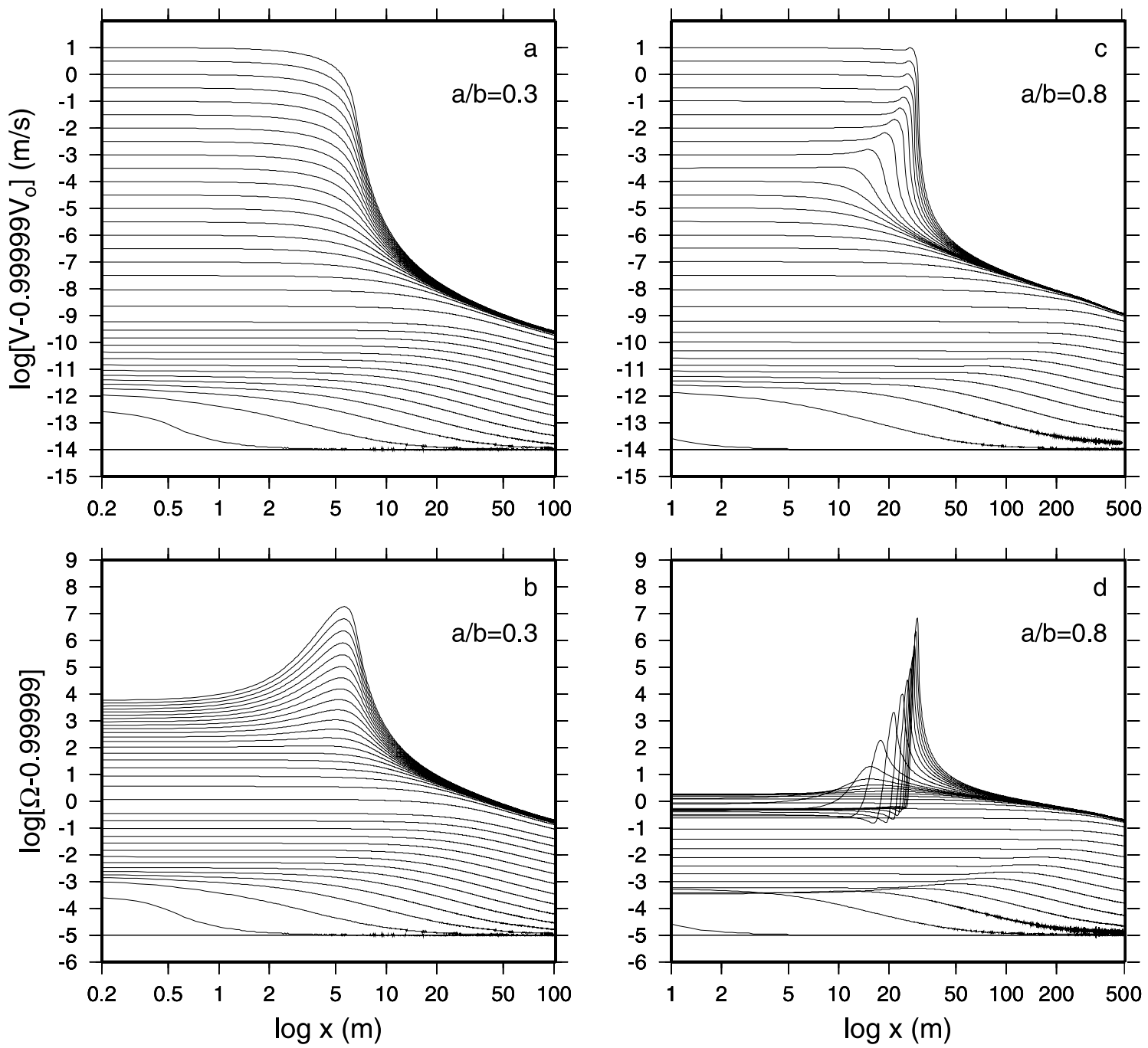

Figure 12. Snapshots of (a and c) $V$ and (b and d) $\Omega$ for a fault that is initially at steady state with $V=$ $10^{-9} \mathrm{~m} / \mathrm{s}$, and for which $\dot{\tau}^{\infty}=0$ except over $|x| \leq 0.1 L_{b-a}$. Figures $12 \mathrm{a}$ and $12 \mathrm{~b}$ are for $a / b=0.3$; Figures $12 \mathrm{c}$ and $12 \mathrm{~d}$ are for $a / b=0.8$ (note the difference in logarithmic horizontal scales). To highlight the early expansion phase, $99.999 \%$ of the initial values are subtracted.

expansion can also be rationalized from a linear perturbation analysis of equations (1) through (3) [e.g., Ruina, 1983]. Ruina's equation (28) for a spring-block slider shows that for small departures from steady state sliding the growth rate of an instability increases as the spring stiffness decreases. Intuitively this makes sense: Smaller stiffnesses lead to smaller reductions in driving stress for a given displacement, and hence to larger growth rates. For an elastically deformable fault, the stiffness of a sinusoidal perturbation in slip is inversely proportional to the wavelength of that perturbation. Having the longest wavelengths grow fastest implies that an initially localized nucleation zone expands with time.

[60] It is also evident that neglect of elasticity promotes localization, because in this case each point along the fault accelerates to instability in accordance with its initial velocity. Equation (17), accurate in the no-healing limit, shows that as the spring stiffness $k$ tends to zero the time of instability is given by $t^{*}=(a / b)\left(D_{c} / V_{o}\right)$, where $V_{o}$ is the initial slip speed. Because $t^{*}$ decreases as $V_{o}$ increases, the ratio of the velocities at any two points with differing velocities diverges with time; i.e., localization occurs. This also explains why the simulations with the least heterogeneity (squares in Figure 2) have the smallest $L_{\min }$ for $a / b>$ 0.5 : Because the differences in velocity as the fault passes through steady state are small, there is more time for the entire surface to evolve (with increasing $\Omega$; see section 5.2) before significant velocity variations arise. This in turn leads to larger values of $\Omega$ upon localization, which favors the no-healing fixed length solution.

[61] In Appendix B we derive self-similar solutions in the no-healing limit that shed some light on these early stages of nucleation. We show that neglect of elasticity, which formally entails dropping terms of order $L_{b} / L$, leads to localization. Neglect of elasticity is appropriate when along-strike gradients in velocity are small (the elastic stressing rate must be small compared to the direct and evolution effects $a \dot{V} / V$ and $b \dot{\theta} / \theta$; see equation (9)). The resulting self-similar solution accurately describes the localization phase in Figure 12, despite the fact that this 
simulation retains elasticity and the full evolution equation $\dot{\theta}=1-\Omega$ (Figure B1). However, a linear perturbation analysis that retains elasticity leads to expansion of the nucleation zone for arbitrary $L_{b} / L$, as suggested by the results of Ruina [1983]. By comparing the neglected nonlinear terms in the expanding solution to the neglected elastic term in the localizing solution, we show that the transition between the two is expected when $L v_{o} \approx L_{b}$, where $v_{o}$ is the magnitude of the velocity perturbation normalized by the background velocity. Physically this makes some sense: Increasing $L$ for the same $v_{o}$ diminishes the tendency for expansion by decreasing the strength of elastic interactions, while increasing $v_{o}$ at the same $L$ (stiffness) promotes localization by increasing (in a superlinear fashion) the rate at which the perturbation velocity diverges from the background velocity.

\subsection{Early Evolution of $\boldsymbol{\Omega}$}

[62] When elasticity can be neglected (or, more precisely, when $\left.\dot{\tau}^{\infty}+\dot{\tau}^{e l} \ll a \dot{V} / V\right)$, the evolution of $\Omega$ can be determined analytically. Equation (9) becomes (independent of the evolution law)

$$
a \frac{\dot{V}}{V}=-b \frac{\dot{\theta}}{\theta}
$$

Because $(\dot{V} \theta) /(V \theta)=\dot{V} / V+\dot{\theta} / \theta, \Omega=V \theta / D_{c}$ changes least the closer $\dot{V} / V$ and $\dot{\theta} / \theta$ approach each other in magnitude, or, from (50), the closer $a$ is to $b$. Formally, integrating (50) yields

$$
\frac{\Omega(t)}{\Omega(0)}=\left(\frac{V(t)}{V(0)}\right)^{\left(1-\frac{a}{b}\right)}
$$

This implies, for example, that to increase $\Omega$ from steady state to 10 requires only a 2 order-of-magnitude increase in $V$ for $a / b=0.5$, but a 10 order-of-magnitude increase for $a / b=0.9$. The upper dashed lines in Figure 3 shows the slope of $(1-a / b)$ predicted by $(51)$; they approximate quite well the increase of $\Omega$ leading to localization for both the localized load on an otherwise homogeneous surface (solid circles) and the small-heterogeneity simulation (open squares). At least in the latter case this is not surprising; reducing velocity variations along the fault minimizes the elastic stressing rate. Further reductions in initial heterogeneity could presumably lead to values of $L_{0.1}$ approaching $L_{v}$ for arbitrary $a / b$.

[63] As localization proceeds, the assumption $L \gg L_{b}$ is progressively violated and elasticity again becomes important; eventually this precipitates the transition from localization to localized acceleration in Figure 12. For a constant stiffness spring-block slider in the no-healing limit, the evolution of $\Omega$ can again be determined analytically. The condition for $\Omega$ to increase with $V$ is $C_{1}>1$, with $C_{1}$ given by (17). Approximating the stiffness by $\mu^{\prime} / 2 L_{\nu}$ and using (21), this condition becomes

$$
\frac{L}{L_{\nu}} \gtrsim \frac{b}{b-a}
$$

This implies, for example, that the transition from increasing to decreasing $\Omega$ occurs at $L \sim 2 L_{\nu}$ for $a / b=$
0.5 , but at $L \sim 10 L_{\nu}$ for $a / b=0.9$. Indeed, for all the simulations in Figures 3 and 9 the peak $\Omega$ is reached while $L$ decreases, consistent with the idea that increasing the stiffness (decreasing $C_{1}$ ) increases the magnitude of $\dot{\theta} / \theta$ relative to $\dot{V} / V$ (equation (23)). Also, although (52) sometimes overestimates and sometimes underestimates the value of $L / L_{v}$ at the peak $\Omega$, we find in our simulations that for $a / b$ from 0.5 to 0.9 it is almost always accurate to within a factor of 2 and usually accurate to within several tens of percent. Taken together, then, equations (51) and (52) provide some rationalization for the numerical result that the peak value of $\Omega$ reached early in the nucleation process decreases with increasing $a / b$ (e.g., Figures 1 and 3).

\section{Summary and Conclusions}

[64] We have obtained analytic estimates of the size of the nucleation zone on faults obeying Dieterich's "aging" version of the rate-and-state friction laws. The more robust of these estimates, applicable in the no-healing limit $(\Omega \equiv$ $\left.V \theta / D_{c} \gg 1\right)$, is that the nucleation zone spontaneously evolves toward a separable solution for accelerating slip on a crack of fixed half length $L_{v}=1.3774 L_{b}$, where $L_{b} \equiv$ $\mu^{\prime} D_{c} / b \sigma$. This is the separable solution for which $K=0$, as is required for the crack tips to remain stationary on an infinite fault without large stress barriers. For $a / b$ less than the critical value of $\sim 0.3781, \Omega$ increases everywhere and the fixed length solution is followed until elastodynamics becomes important. For $a / b>0.3781, \Omega$ at the center of the nucleation zone decreases as instability is approached and eventually the no-healing limit becomes inapplicable. The decrease in $\Omega$ is slow for $a / b$ only slightly larger than critical, so for even modest values of $\Omega$ the nucleation zone can reach seismic velocities before departing significantly from the fixed length solution. Large initial values of $\Omega$ are the reason many of the examples shown by Dieterich [1992] follow this solution even for moderately large $a / b$.

[65] Although in the no-healing limit the numerical simulations tend to the fixed length solution for which $K=0$, this is not the smallest patch capable of reaching instability. Separable solutions for shorter patches have $K>0$, and so would not arise spontaneously unless the ends of the nucleation zone were somehow pinned. The minimum viable nucleation length decreases with decreasing $a / b$ and for $a=0$ is identical to the universal nucleation length for slip-weakening faults derived by Uenishi and Rice [2003]. Although for $a=0$ our governing equations are identical to slip-weakening friction only in the no-healing limit, in practice all our simulations with small $a / b$ evolve to this limit.

[66] For $a / b>0.3781, \Omega$ in the interior of the nucleation zone decreases to a quasi-constant value $\Omega$ modestly in excess of 1 , and the nucleation zone takes on the appearance of an expanding slip-weakening crack. By balancing the stress drop in the interior (constant $\Omega$ ) with the effective slip-weakening properties at the crack tip $(\Omega \gg 1)$, we find that the size of the nucleation zone approaches $L_{\infty}=\pi^{-1}[b /$ $(b-a)]^{2} L_{b}$ in the limit that $V$ is many orders of magnitude larger than background. This value is approached reasonably closely in most of our simulations by the time the slip velocity reaches $\sim 0.1 \mathrm{~m} / \mathrm{s}$, which is roughly when elasto- 
dynamics would become important. The associated estimate for the limiting value of $\Omega$ is $[1-(\pi / 2)(b-a) / b]^{-1}$. In fact, for reasons we do not understand, the simulations often closely approach this value of $\Omega$ well before the nucleation length reaches $L_{\infty}$.

[67] For a spring-block slider to maintain a constant $\Omega$ during the approach to instability requires a unique spring stiffness $k=\sigma(b-a)\left(1-\Omega^{-1}\right) / D_{c}$. The effective stiffness in the simulations, as measured by $-\dot{\tau} / V$, tends to oscillate around this value, with the result that $\Omega$ is only approximately constant. As these oscillations increase in amplitude the nucleation process appears increasingly complex, with, in extreme cases, the nucleation zone alternately decelerating when $\Omega<1$ and approaching the no-healing fixed length solution when $\Omega \gg 1$. For a given $a / b$ the oscillations tend to become larger with increasing slip velocity, and large oscillations begin at smaller velocities as $a / b$ approaches 1 . For $a / b \gtrsim 0.9$ and our adopted loading conditions these complications begin at slip velocities less than $0.1 \mathrm{~m} / \mathrm{s}$, giving rise to the scatter seen in Figure 8 for a subset of the simulations.

[68] Laboratory experiments indicate that even for velocity-weakening surfaces $a / b$ commonly exceeds 0.9 [e.g., Kilgore et al., 1993; Blanpied et al., 1998]. In addition, in a crust (or numerical model) where the constitutive properties vary only with temperature, $a / b$ is essentially guaranteed to be close to 1 near the creeping/locked (velocity weakening/ velocity strengthening) transition. In numerical models these are regions of large stressing rate and preferential earthquake nucleation [Lapusta and Rice, 2003]. That $L_{\infty}$ scales as $\sim b /(b-a)^{2}$ indicates that the nucleation zone can be much larger than Dieterich's [1992] estimate under these conditions. To the extent that such nucleation zones are sensitive to relatively long-wavelength properties of the fault surface, the spatial variation of $(b-a)$ can be expected to play an important role during nucleation in these regions as well. Thus nucleation with $a / b$ near 1 is likely to be both geologically relevant and quite complex. As advocated by Lapusta and Rice [2003] it may be important to run simulations over multiple seismic cycles in these cases so as to remove a degree of arbitrariness in the initial conditions (Figures 2 and 8 show that this is not an issue for low-to-moderate values of $a / b$ ).

[69] The large disparity between the length scales $L_{\nu}$ and $L_{\infty}$ for $a / b$ near 1 has interesting implications for the possible detectability of earthquake nucleation phases. Temporary excursions of the fault surface to modest values of $\Omega$ can cause cycling between these length scales and large variations in moment acceleration that are reminiscent of the hesitant beginnings of earthquakes observed by Ellsworth and Beroza [1995, 1998]. For this to be detectable using surface seismometers appears to require large values of both $a / b(\gtrsim 0.95)$ and $D_{c}$ (at least $\left.100 \mu \mathrm{m}\right)$. Magnitude $>4$ "nucleation phases" [Ellsworth and Beroza, 1995], as well as occasional hours-long foreshock sequences that may be indicative of an underlying kilometer-scale nucleation process [Dodge et al., 1996], seem to fall outside the realm of the rate-and-state equations discussed here, at least with anything approaching lab values of the parameter $D_{c}$. There are some lingering questions regarding the scaling of $D_{c}$ to natural faults, however. Marone and Kilgore [1993] suggest that in the presence of gouge $D_{c}$ might reach $1 \mathrm{~mm}$, and Scholz [1988] points out that polished fault surfaces in the laboratory differ from natural (fractal) faults in that they possess a characteristic contact dimension (he suggests that $D_{c}$ in the seismogenic zone might be as large as $10 \mathrm{~mm}$ ). For values in excess of $1 \mathrm{~mm}$, equation (49) indicates that the "bang" marking the transition from nucleation to propagation might be detectable with sufficiently sensitive surface instruments even for "simple" nucleation as in Figure 11a (that is, even without the cycling between $L_{v}$ and $\left.L_{\infty}\right)$.

[70] Given that our adopted evolution law is essentially empirical, it is appropriate to ask if it is relevant to natural faults. In particular, our estimate of $L_{\infty}$, as well as previous estimates of the effective slip-weakening distance for dynamic ruptures, make use of the prediction of the aging law that following a large velocity jump the slip-weakening distance is larger than $D_{c}$ by the logarithm of the ratio of the velocities. This property is not shared by the slip law. Moreover, both laws appear to have been crafted to explain the surface evolution during slide-hold-slide tests and velocity-stepping tests which together encompass the range $\Omega \ll 1$ and $0.1 \lesssim \Omega \lesssim 10$. Our results show that the surface evolution in the range $\Omega \gg 1$ is crucial in determining the fracture energy and hence nucleation size, at least in the constant-weakening limit. Further experimental work with a larger range of velocity steps would thus seem warranted. Finally, a large $L_{\infty}$ would require that the nucleation we have described could survive short circuiting by additional weakening processes, such as thermal pressurization of pore fluid [e.g., Segall and Rice, 2004]. It is hoped that the approach followed here, which basically entails balancing the weakening rate in the interior of the nucleation zone with the strengthening rate near the ends, or alternatively balancing the tendency of elasticity to expand the nucleation zone with the tendency of slip or velocity weakening to shrink it, will prove useful even in the face of additional weakening processes or alternate evolution laws.

\section{Appendix A: Scaling Considerations}

\section{A1. Dimensionless Equations}

[71] We can normalize the governing equations using $D_{c}$ as the displacement scale, $\mu^{\prime} D_{c} / b \sigma$ as the spatial coordinate scale, and $b \sigma / \dot{\tau}^{\infty}$ as the time scale. Using tildes to denote dimensionless quantities, equations (1) and (3) become

$$
\begin{gathered}
\frac{a}{b} \frac{\tilde{\dot{V}}}{\tilde{V}}+\frac{\tilde{\dot{\theta}}}{\tilde{\theta}}=1+\frac{1}{2 \pi} \int_{-\infty}^{\infty} \frac{d \tilde{V} / d \tilde{s}}{\tilde{s}-\tilde{x}} d \tilde{s}, \\
\tilde{\dot{\theta}}=1-\tilde{V} \tilde{\theta},
\end{gathered}
$$

with initial conditions

$$
\begin{gathered}
\tilde{V}_{i}=\frac{V_{i} b \sigma}{D_{c} \dot{\tau}^{\infty}}, \\
\tilde{\theta}_{i}=\frac{\theta_{i} \dot{\tau}^{\infty}}{b \sigma} .
\end{gathered}
$$

Thus the dimensionless governing equations depend only upon the ratio $a / b$ and the initial values $\tilde{V}_{i}$ and $\tilde{\theta}_{i}$. Varying $b$ 
in the dimensional equations at the same $a / b$, as in Figures 2 and 8 , is equivalent, after suitably scaling the spatial and temporal scales, to varying $V_{i} \sigma / \dot{\tau}^{\infty}$ and $\sigma / \dot{\tau}^{\infty} \theta_{i}$ by the same factor instead.

\section{A2. One-Dimensional Spring-Block Slider Cycles With Radiation Damping}

[72] To estimate the velocity at which dynamics plays a role, we add the radiation damping term $\tau^{\mathrm{rad}}=V \mu / 2 c_{S}$ to the equilibrium equation, where $\mu$ is the shear modulus and $c_{s}$ is the shear wave speed. This term accounts for how the local fault impedance relates slip velocities to stress, as a onedimensional (1-D) strain wave propagates into the host rock. It does not account for stress transfer between different points on the fault via elastic wave propagation; in this sense radiation damping can be thought of as true elastodynamics in the limit of spatially uniform motion of the fault surface. Equation (9) becomes

$$
\frac{\mu}{2 c_{s}} \dot{V}+a \sigma \frac{\dot{V}}{V}+b \sigma \frac{\dot{\theta}}{\theta}=\dot{\tau} .
$$

For $\Omega \gg 1$ (no-healing limit) $\dot{\theta} / \theta=-V / D_{c}$, whereas for $\Omega=$ const (constant-weakening limit) $\dot{\theta} / \theta=-\dot{V} / V$. Substituting these into (A5) and rearranging yields

$$
\begin{gathered}
\dot{V}=\frac{\dot{\tau}+b \sigma \frac{V}{D_{c}}}{\frac{\mu}{2 c_{s}}+\frac{a \sigma}{V}}, \quad \Omega \gg 1 ; \\
\dot{V}=\frac{\dot{\tau}}{\frac{\mu}{2 c_{s}}+\frac{(a-b) \sigma}{V}}, \quad \Omega=\text { const } .
\end{gathered}
$$

As $\dot{\tau}$ depends upon $V$ but not $\dot{V}$, these equations show that radiation damping begins to dominate the solution for $V>$ $V_{\text {dyn }}$, where

$$
V_{\mathrm{dyn}}=2 \frac{a \sigma}{\mu} c_{s}, \Omega \gg 1 ; \quad V_{\mathrm{dyn}}=2 \frac{(a-b) \sigma}{\mu} c_{s}, \Omega=\text { const. }
$$

If one adds another requirement for the applicability of quasi-static elasticity, that the slip velocity changes little (say by less than a factor of 2) in the time it takes shear waves to traverse the nucleation half length $L$, then from equations (21) and (45) (for $\Omega \gg 1$ ) or (42) and (44) (for $\Omega=$ const), the expressions for $V_{\text {dyn }}$ are identical except that they lack the leading coefficient of 2 . For reasonable parameters $V_{\text {dyn }}$ is of order $0.1 \mathrm{~m} / \mathrm{s}$.

[73] For moderately large values of $a / b$ it is appropriate to ask how closely the nucleation length approaches $L_{\infty}$ before $V_{\text {dyn }}$ is reached. In this regard it seems relevant to determine the velocity at which nucleation zones on natural faults cross the steady state curve, because with $V_{\text {dyn }}$ fixed this determines the available velocity range of nucleation. To address this issue, we model the earthquake cycle using a 1-D spring-block slider system with spring constant $k$ and radia1378 tion damping (no inertia). Equation (A5) then becomes

$$
\frac{\mu}{2 c_{s}} \dot{V}+a \sigma \frac{\dot{V}}{V}+b \sigma \frac{\dot{\theta}}{\theta}=k\left(V_{p l}-V\right),
$$

where $V_{p l}$ is the load point velocity. We assume velocity weakening behavior $(b>a)$ and subcritical stiffness $\left(k<k_{c}\right.$,
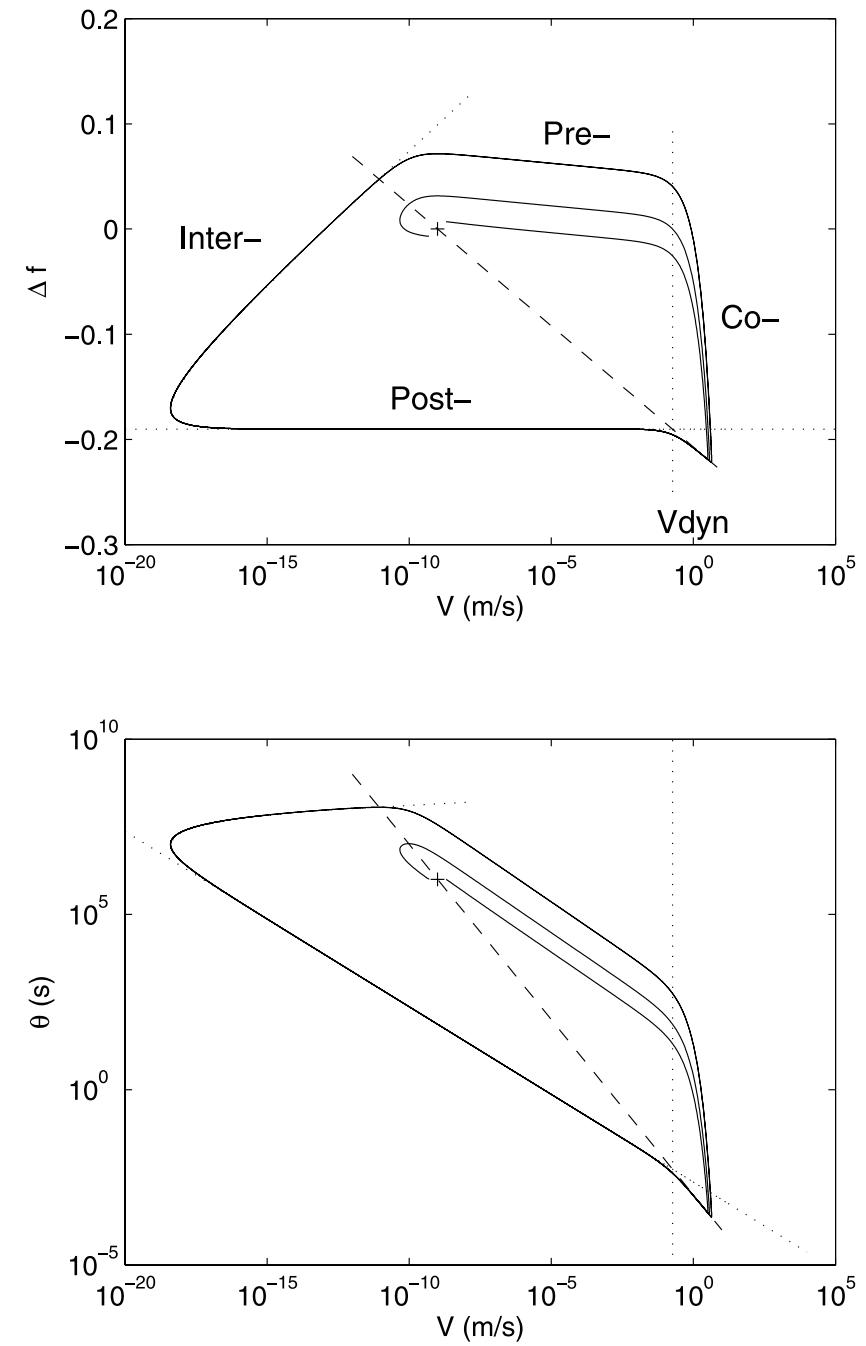

Figure A1. Typical 1-D cycle. Parameters are $a=1 \%, b=$ $2 \%, D_{c}=1 \mathrm{~mm}, c_{s}=3 \mathrm{~km} / \mathrm{s}, \mu=32.4 \mathrm{GPa}, \sigma=100 \mathrm{MPa}$, $k=0.2 * k_{c}, V_{p l}=10^{-9} \mathrm{~m} / \mathrm{s}$. Trajectories for two different initial conditions are shown: $V(0)=0.5 V_{p l}$ and $V(0)=2 V_{p l}$, both with $\theta(0)=V_{p l} / D_{c}$. Both cases converge to a common limit cycle. (top) Variation in friction and (bottom) state variable. Different stages of the cycle are labeled in Figure A1 (top). $V_{\text {dyn }}$ is the slip rate above which radiation damping becomes important. The dashed line indicates steady state; the dotted trajectory the approximation for the postseismic and interseismic periods from (A12).

where $\left.k_{c}=\sigma[b-a] / D_{c}\right)$. Figure A1 shows typical numerical results and defines the different stages of the cycle.

\section{A2.1. Postseismic and Interseismic Phases}

[74] At the end of a dynamic event the fault returns to steady state, so the postseismic stage starts with $V=V_{\text {dyn }}$ and $\theta=\theta_{\text {dyn }}=D_{c} / V_{\text {dyn }}$. For $D_{c}$ of order $1 \mathrm{~mm}, \theta_{\text {dyn }}$ is a short time of order $1 \mathrm{~ms}$. The postseismic and interseimic stages are dominated by healing, with $V \theta / D_{c} \ll 1$ and $\theta \approx 1$. As $k<$ $k_{c}$ and $b /(b-a)>1, V \theta / D_{c} \ll 1$ implies $k V \ll \sigma b / \theta$, so the elastic stressing rate can be neglected, as can radiation damping. The governing equations become

$$
a \sigma \frac{\dot{V}}{V}+b \sigma \frac{\dot{\theta}}{\theta}=k V_{p l}
$$


with $\theta=\theta_{\text {dyn }}+t$ (by convention the postseismic stage starts at $t=0)$. The solution is

$$
V=V_{\mathrm{dyn}}\left(\frac{\theta_{\mathrm{dyn}}+t}{\theta_{\mathrm{dyn}}}\right)^{-b / a} \exp \left(\frac{k V_{p l} t}{a \sigma}\right),
$$

which we rewrite as

$$
V=V_{\mathrm{dyn}}\left(\frac{\exp \left[t /\left(t_{p}+\theta_{\mathrm{dyn}}\right)\right]}{1+t / \theta_{\mathrm{dyn}}}\right)^{b / a},
$$

where $t_{p}=b \sigma /\left(k V_{p l}\right)-\theta_{\text {dyn }}$ is the characteristic duration of the postseismic stage, defined as the turning point time $(\dot{V}=$ $0)$. Typically $t_{p} \approx b \sigma /\left(k V_{p l}\right)$, and in our example $t_{p}=10^{7} \mathrm{~s}$ (116 days). This solution is plotted in Figure A1 as a dashed curve.

[75] When $t \ll t_{p}$ the fault slows down at nearly constant shear stress, with a power law decay in time:

$$
V(t) \approx \frac{V_{\mathrm{dyn}}}{\left(t / \theta_{\mathrm{dyn}}\right)^{b / a}} .
$$

This behavior differs from that of a spring-block slider with inertia and no radiation damping, which undergoes a large stress decrease associated with overshoot in the early postseismic stage [Rice and Tse, 1986]. Later, during the interseismic stage, $V$ grows quasi-exponentially:

$$
V(t) \approx V_{\mathrm{dyn}}\left(\frac{e^{t / t_{p}}}{t / \theta_{\mathrm{dyn}}}\right)^{b / a} .
$$

[76] The preseismic (nucleation) stage starts when steady state is reached. From the numerical results it seems that the previous solution can be extended up to that point. Taking $t \approx \theta=D_{c} / V_{s s}\left(\approx 10^{8} \mathrm{~s}\right.$ or 3 years in our example $)$, the slip rate $V_{s s}$ at the transition is such that:

$$
V_{s S}=V_{\mathrm{dyn}}\left(\frac{e^{D_{c} /\left(V_{s s} t_{p}\right)}}{V_{\mathrm{dyn}} / V_{s s}}\right)^{b / a} .
$$

Defining $\xi=k V_{p l} / k_{c} V_{s s}$ and $\xi=k V_{p l} / k_{c} V_{\text {dyn }}$, that equation reads $\xi e^{-\xi}=\xi_{0}$. As $\xi_{0} \ll 1$, the solution has the asymptotic expansion $\xi \approx-\ln \left(\xi_{0}\right)+\ln \left(\ln \left(\xi_{0}\right)\right)$ [Hinch and Crighton, 1991]. To first order, the slip rate at the interseismic to preseismic transition is given by

$$
\frac{V_{s s}}{V_{p l}} \approx \frac{k / k_{c}}{\ln \left(\frac{k_{c}}{k} \frac{V_{\mathrm{dyn}}}{V_{p l}}\right)} .
$$

The maximum $V_{s s} / V_{p l}$ occurs for $k \approx k_{c}$, and for $V_{\mathrm{dyn}} \approx$ $0.1 \mathrm{~m} / \mathrm{s}$ and $V_{p l} \approx 10^{-9} \mathrm{~m} / \mathrm{s}$ this equals $\sim 1 / 20$. For the example in Figure A1, with $k / k_{c}=0.2, V_{s s} \approx 10^{-11} \mathrm{~m} / \mathrm{s}$, 2 orders below $V_{p l}$.

[77] As usual, there is inherent ambiguity in transferring these results to an elastically deformable fault, but rearranging (A16) shows dependence upon the ratios of $k V_{p l}, k_{c} V_{\text {dyn }}$, and $k_{c} V$. Of these variables only the time-dependent fault stiffness $k$ requires clarification, and that it occurs only in the product $k V_{p l}$ shows that it is the loading rate during the postseismic and interseismic intervals that is important. For a dynamic rupture of length $L$ that was previously locked, a reasonable maximum average loading rate is $\sim V_{p l} \mu^{\prime} / L$, implying a stiffness of $\mu^{\prime} / L$. Thus it seems acceptable to view $k_{c} / k$ as the ratio of the rupture size to the minimum nucleation size for unstable slip, suggesting that the slip velocity upon first crossing the steady state line following the interseismic period is a few orders of magnitude below $V_{p l}$. In a gross sense this is consistent with our simulations.

\section{A2.2. Preseismic Phase}

[78] Equation (A16) is the main result of the springblock-slider discussion; in the remainder we merely close the cycle. Nucleation begins when the fault passes through steady state, but for $k$ several times smaller than $k_{c}$ most of the nucleation phase takes place well above steady state with $V<V_{\text {dyn }}$. This is the (no-healing) approximation of Dieterich [1994]. Substituting $\dot{\theta}=-V \theta / D_{c}$ into (A9) and dropping radiation damping, the solution is

$$
V(t)=\frac{V(0)}{1+\left(1+\frac{V(0)}{V_{p l}}\left(1-\frac{\sigma b}{k D_{c}}\right)\right)\left(e^{t / t_{a}}-1\right)},
$$

with $t_{a}=a \sigma / K V_{p l}$ a loading timescale.

\section{A2.3. Coseismic Phases}

[79] During fast sliding $\left(V>V_{\text {dyn }}\right)$ we can neglect the direct effect and the tectonic loading rate. We find that the slip rate during the two coseismic phases, acceleration and arrest, behaves roughly exponentially (see Figure A2).

[80] During the acceleration phase, we neglect healing:

$$
\left(\mu / 2 c_{s}\right) \dot{V}-\sigma b V / D_{c}=-k V .
$$

This leads to

$$
V \propto \exp \left(\frac{\sigma b / D_{c}-k}{\mu / 2 c_{s}} t\right)
$$

As $\theta \propto \exp \left(-\delta / D_{c}\right)$ in the no-healing approximation, the state falls superexponentially and the fault comes back to steady state $\left(\theta V / D_{c} \rightarrow 1\right)$.

[81] During the arrest phase, we assume steady state $\left(V \theta / D_{c}=1\right)$ :

$$
\left(\mu / 2 c_{s}\right) \dot{V}=-k V \text {, }
$$

which leads to

$$
V \propto \exp \left(-\frac{k}{\mu / 2 c_{s}} t\right)
$$

\section{Appendix B: Self-Similar Solutions for Expansion and Contraction of the Nucleation Zone}

[82] Here we develop self-similar solutions, in the limit that $\Omega \equiv V \theta / D_{c} \gg 1$, for the early expansion and localization stages of nucleation seen in Figure 12. In fact, in the nohealing limit the velocity distributions in the two cases are identical. By comparing the magnitude of the terms that are 

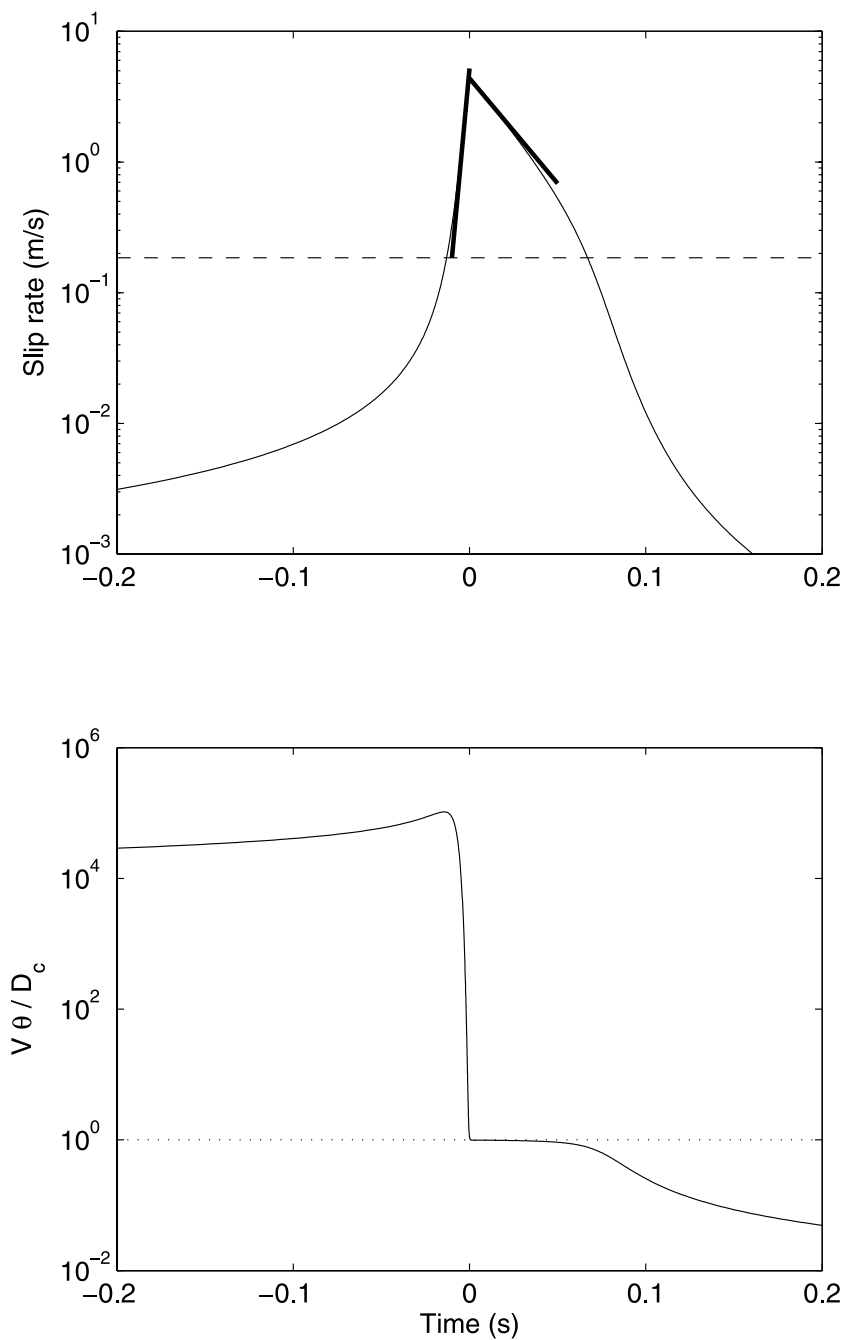

Figure A2. Zoom on the coseismic phase. Time is referenced to the time of maximum slip rate. (top) Slip rate $V(t)$. The dashed line is the dynamic threshold $V_{\text {dyn }}$. Bold lines show the two exponential phases, acceleration and arrest. (bottom) The $\theta V / D_{c}$ initially far above steady state (dotted line). In the acceleration phase the drop of $\theta$ overcomes the growth of $V$ and steady state is approached. During dynamic arrest steady state is maintained.

dropped in the two derivations we identify the criterion that determines the transition from expansion to localization.

[83] We begin by seeking a solution of the form

$$
V(x, t)=V_{o}(t)\left[1+v_{o}(t) \mathcal{V}\left(\frac{x}{L(t)}\right)\right],
$$

where the perturbation $v(x, t)=v_{o}(t) \mathcal{V}(x / L)$ grows on top of a uniform background solution $V_{o}(t)$, and the length scale $L$ may increase or decrease with time. The dimensionless velocity distribution $\mathcal{V}$ is normalized such that $\mathcal{V}(0)=1$, so $v_{o} V_{o}$ is the magnitude of the velocity perturbation at the origin. Self-similarity implies (writing $\xi$ for $x / L$ )

$$
\dot{v}=\dot{v}_{o} \mathcal{V}-v_{o} \mathcal{V}^{\prime} \xi \frac{\dot{L}}{L}
$$

where $\mathcal{V}^{\prime}=d \mathcal{V} / d \xi$. The corresponding stressing rate is

$$
\dot{\tau}^{e l}=V_{o} v_{o} \frac{\mu^{\prime}}{2 L} \mathcal{H}\left[\mathcal{V}^{\prime}\right]
$$

In the following we define $L$ such that $\mathcal{H}\left[\mathcal{V}^{\prime}\right](0)=-1$.

[84] Adopting the no-healing limit $\Omega \gg 1$, substituting (B1) - (B3) into (10) yields

$$
\begin{gathered}
a\left[\frac{\dot{V}_{o}}{V_{o}}+\frac{\dot{v}_{o} \mathcal{V}-v_{o} \mathcal{V}^{\prime} \xi \dot{L} / L}{1+v_{o} \mathcal{V}}\right]-\frac{b}{D_{c}} V_{o}\left[1+v_{o} \mathcal{V}\right] \\
\quad=V_{0} v_{o} \frac{\mu^{\prime}}{2 \sigma L} \mathcal{H}\left[\mathcal{V}^{\prime}\right]
\end{gathered}
$$

Far from the perturbation $\mathcal{V}, \mathcal{V}^{\prime}$, and $\mathcal{H}$ tend to zero so the background solution obeys

$$
\frac{\dot{V}_{o}}{V_{o}^{2}}=\frac{b}{a D_{c}},
$$

with solution

$$
V_{o}=V_{o}(0)\left(1-\frac{t}{t^{*}}\right)^{-1}
$$

so once again the slip velocity increases as the inverse of the time to instability, where the time of instability $t^{*}=$ $a D_{c} / b V_{o}(0)$. Substituting (B5) into (B4) yields

$$
\frac{a D_{c}}{b V_{o} v_{o}}\left[\frac{\dot{v}_{o} \mathcal{V}-v_{o} \mathcal{V}^{\prime} \xi \dot{L} / L}{1+v_{o} \mathcal{V}}\right]=\mathcal{V}+\frac{L_{b}}{2 L} \mathcal{H}\left[\mathcal{V}^{\prime}\right]
$$

which is the starting point for both the expanding and localizing solutions to follow.

\section{B1. Neglect of Elasticity Leads to Localization}

[85] Noting that $\mathcal{V}$ and $\mathcal{H}$ are of order 1 in the region of interest, it appears that for $L \gg L_{b}$ we can neglect elastic interaction and (B7) becomes

$$
\frac{a D_{c}}{b V_{o} v_{o}}\left[\frac{\dot{v}_{o} \mathcal{V}-v_{o} \mathcal{V}^{\prime} \xi \dot{L} / L}{1+v_{o} \mathcal{V}}\right]=\mathcal{V}
$$

At $\xi=0(\mathcal{V}=1)$ this becomes

$$
\frac{\dot{v_{o}}}{v_{o}\left(1+v_{o}\right)}=\frac{b V_{o}}{a D_{c}}
$$

with solution

$$
v_{o}(t)=\frac{v_{o}(0)}{\left(1+v_{o}(0)\right)\left(1-t / t^{*}\right)-v_{o}(0)}=\frac{v_{o}(0)}{1-t / t_{0}^{*}},
$$

where $t_{0}^{*}=a D_{c} / b V(0)=t^{*} /\left(1+v_{o}(0)\right)$ is the time of instability at the origin. As $t^{*}<t^{*}$, the perturbation reaches instability sooner than the uniform background motion.

[86] Inserting (B9) into (B8) leads to

$$
\frac{\mathcal{V}(1-\mathcal{V})}{\xi \mathcal{V}^{\prime}}=\frac{\dot{L}}{L} \frac{1+v_{o}}{\dot{v}_{o}}
$$



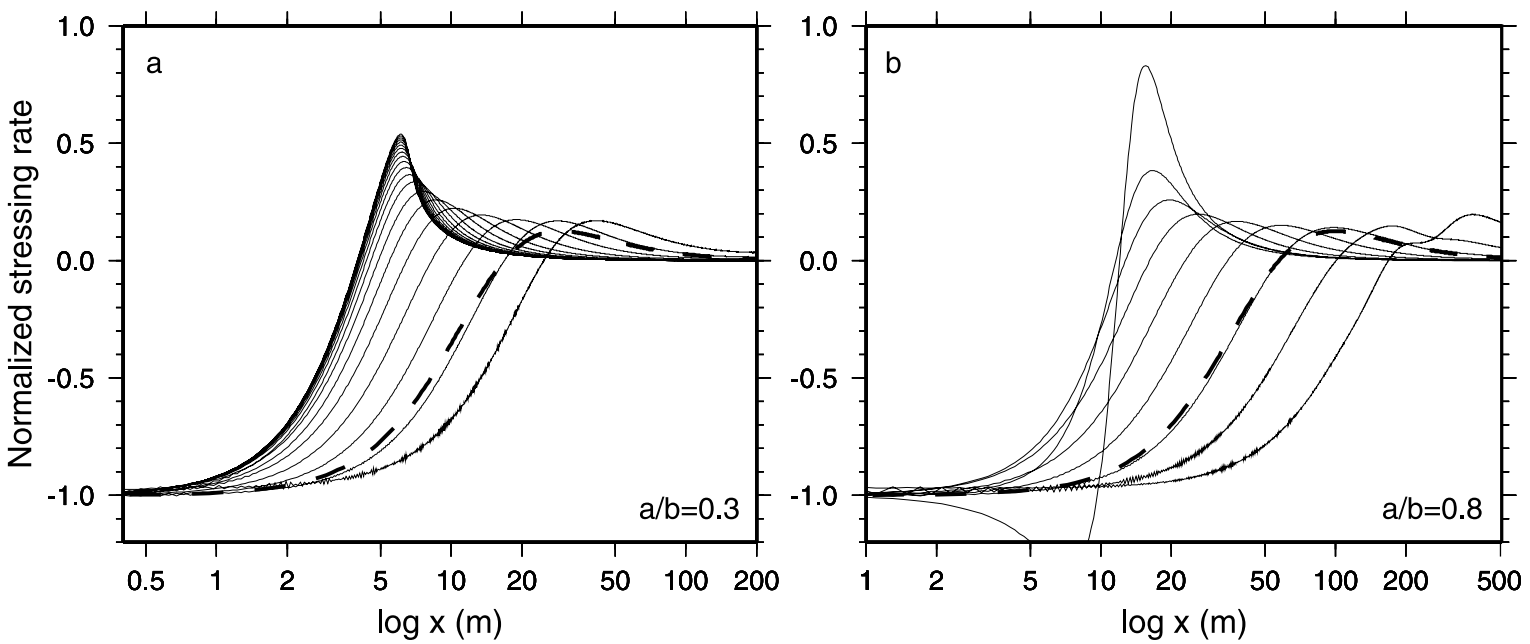

Figure B1. Snapshots of the elastic stressing rate, normalized by that at the origin, for the simulations of Figure 12. For $a / b=0.3$ (a), all snapshots following the onset of localization are shown. For $a / b=0.8(b)$, snapshots are shown from the onset of localization to roughly when $L_{\min }$ is reached. The bold dashed curves indicate the no-healing, no-elasticity self-similar distribution from equation (B17). The agreement is better for $a / b=0.8$, even though $V \theta / D_{c}$ during localization is only $\sim 2$, perhaps because $L / L_{b}$ is not as large for $a / b=0.3$ (although we note also that when elasticity is neglected, one can show that in the limit that $a$ approaches $b$ the self-similar distributions of (B16) and (B17) arise even without the no-healing approximation).

For a function of position (on the left) to equal a function of time (on the right), both must be constant. Because $0<\mathcal{V} \leq$ 1 and $\mathcal{V}^{\prime}$ is maximal at $\xi=0$, this constant is negative and $L$ decreases with time. Writing this constant as $-(1 / n)$ and using (B9), we have

$$
\frac{\dot{L}}{L}=-\frac{1}{n} \frac{b}{a D_{c}} V_{o} v_{o}
$$

Making use of (10) with $\dot{\tau}$ set to zero, this has the solution

$$
L(t)=L(0)\left(\frac{1+v_{o}(0)}{1+v_{o}}\right)^{1 / n} .
$$

[87] To determine the shape of the velocity perturbation, again from (B11) we have

$$
\frac{\mathcal{V}(1-\mathcal{V})}{\mathcal{V}^{\prime} \xi}=-\frac{1}{n},
$$

which has the solution

$$
\mathcal{V}=\left(1+\alpha|\xi|^{n}\right)^{-1} .
$$

The coefficient $\alpha$ is fixed by the definition of the length scale $L$. However, in the absence of additional information there seems to be no mechanism for favoring one value of $n$ over another. Indeed, numerical simulations with elastic interactions turned off (and employing $\dot{\theta}=-\Omega$ ) show that initial velocity distributions of $\left(1+|\xi|^{n}\right)^{-1}$ remain self- similar for arbitrary $n$. However, we find that simulations that include elasticity (and sufficiently weak heterogeneity) spontaneously evolve while $L \gg L_{b}$ toward a velocity distribution of $\left(1+\xi^{2}\right)^{-1}$ for a variety of initial conditions, including initial conditions of the form of (B15) with $n$ different from 2. For $n=2$ the Hilbert transform can be expressed analytically and the normalization condition $\mathcal{H}\left[\mathcal{V}^{\prime}\right](0)=-1$ fixes $\alpha=1$. The resulting slip rate and stressing rate functions are

$$
\mathcal{V}(\xi)=\left(1+\xi^{2}\right)^{-1} ;
$$

$$
\mathcal{H}\left[\mathcal{V}^{\prime}\right](\xi)=-\frac{1-\xi^{2}}{\left(1+\xi^{2}\right)^{2}} .
$$

This slip rate profile is identical to the slip profile that arises during localization on a fault with quadratic slip-dependent friction [Suo et al., 1992]. Figure B1 compares the stressing rate distribution of (B17) to the normalized stressing rates in the simulations shown in Figure 12 (which retain the full evolution equation (3) and elasticity). Self-similarity is indicated by the degree to which the snapshots are just horizontally shifted versions of one another. The agreement between the self-similar and numerical distributions is quite good and is maintained to values of $L$ that are roughly twice the ultimate nucleation length, even when $\Omega$ is only $\sim 2$ (right panel). For smaller $L$, elasticity becomes important and the nucleation zone undergoes a transition from localization to localized acceleration (for large $\Omega$; left panel) or expansion (for $\Omega$ closer to 1 ).

[88] To perhaps gain some insight into the role of elasticity in fixing $n=2$, evaluate the governing equation (B7) 
at $\xi=0$ and substitute that result and (B12) back into (B7) to obtain

$$
\begin{aligned}
\frac{L_{b}}{2 L(t)} & {\left[\frac{\left(1+v_{o}\right) \mathcal{V}}{1+v_{o} \mathcal{V}}+\mathcal{H}+\frac{v_{o} \mathcal{V}^{\prime}}{1+v_{o} \mathcal{V}} \frac{\xi}{n}\right] } \\
= & {\left[\frac{\left(1+v_{o}\right) \mathcal{V}}{1+v_{o} \mathcal{V}}-\mathcal{V}+\frac{v_{o} \mathcal{V}^{\prime}}{1+v_{o} \mathcal{V}} \frac{\xi}{n}\right] . }
\end{aligned}
$$

Because the left side of (B18) is the product of a (nonconstant) function of time alone and a function of position alone (in brackets), and the right is a function of position alone, equality requires that both sides equal zero. The bracketed functions cannot both be zero because this would require that the normalized stressing rate exactly mirror the normalized velocity (that is, $\mathcal{H}=-\mathcal{V}$ ). In the limit of large $L$ the left side approaches zero and (B14) is recovered. Nonetheless, the left side of (B18) is nonzero for finite $L$. We find numerically that the favored solution $(n=$ 2 ) is that which minimizes the maximum value of the bracketed expression on the left; that is, it minimizes the maximum difference between $\mathcal{H}$ and $-\mathcal{V}$. For $n=2$ this maximum occurs at $\xi=1$ and equals $1 / 2$.

\section{B2. Linearization With Elasticity Leads to Expansion}

[89] We next obtain a self-similar solution that retains elasticity by evaluating (B7) in the limit of small velocity perturbations $v_{o}$. This amounts to dropping the $v_{o} \mathcal{V}$ term in the denominator of the bracketed expression on the left, which leads to

$$
\frac{a D_{c}}{b}\left[\dot{v}_{o} \mathcal{V}-v_{o} \mathcal{V}^{\prime} \xi \frac{\dot{L}}{L}\right]-V_{o} v_{o} \mathcal{V}=V_{0} v_{o} \frac{L_{b}}{2 L} \mathcal{H}\left[\mathcal{V}^{\prime}\right]
$$

Maintaining the normalizations $\mathcal{V}(0)=1$ and $\mathcal{H}\left(\mathcal{V}^{\prime}\right)(0)=$ -1 , evaluating $(\mathrm{B} 19)$ at $\xi=0$ yields

$$
\frac{a D_{c}}{b} \dot{v}_{o}-V_{o} v_{o}=-V_{0} v_{o} \frac{L_{b}}{2 L} \text {. }
$$

Substituting this back into (B19),

$$
\frac{-\mathcal{V}-\mathcal{H}\left(\mathcal{V}^{\prime}\right)}{\mathcal{V}^{\prime} \xi}=\frac{2 a \sigma}{\mu^{\prime}} \frac{\dot{L}}{V_{o}}
$$

[90] An analysis using a forward and inverse Fourier transform (not shown here) shows that the self-similar velocity distribution satisfying (B21) is identical to that satisfying (B11) of section B1. So, substituting (B16) for $\mathcal{V}$ and (B17) for $\mathcal{H}$ into (B21) we obtain

$$
\frac{\mathcal{V}(1-\mathcal{V})}{\mathcal{V}^{\prime} \xi}=-\frac{a \sigma}{\mu^{\prime}} \frac{\dot{L}}{V_{o}}=-\frac{1}{2}
$$

which leads to

$$
\dot{L}=\frac{\mu^{\prime}}{2 a \sigma} V_{o}=\frac{\mu^{\prime}}{2 a \sigma} V_{o}(0)\left(1-t / t^{*}\right)^{-1},
$$

where $t^{*}=a D_{c} / b V_{o}(0)$ is the time of instability for the background solution. Integration yields

$$
L(t)=-\frac{L_{b}}{2} \ln \left(1-t / t^{*}\right)+L(0) .
$$

Note that in this case $L$ increases with time, albeit slowly compared to the decrease during localization (in the limit that $t \ll t^{*}$ and $v_{o} \ll 1,\left|\mathrm{~d} L / \mathrm{d}\left(\ln V_{o}\right)\right|$ during localization exceeds that during expansion by roughly the ratio $L / L_{b}$ ).

[91] To determine $v_{o}$, evaluating (B19) at $\xi=1(\mathcal{V}=1 / 2$; $\mathcal{H}\left(\mathcal{V}^{\prime}\right)=0 ; \mathcal{V}^{\prime}=-1 / 2$ ) yields

$$
\frac{\dot{v}_{o}}{v_{o}}=\frac{b V_{o}}{a D_{c}}-\frac{\dot{L}}{L},
$$

which has the solution

$$
v_{o}(t)=L^{-1} v_{o}(0)\left(1-t / t^{*}\right)^{-1} .
$$

\section{B3. Expansion or Localization?}

[92] The above results present something of a paradox. As (B22) is identical to (B14) (since in the latter $n=2)$, the velocity distributions $\mathcal{V}$ for the expanding and localizing solutions are identical. Given this velocity distribution as an initial condition, then, which solution does a numerical simulation follow? To address this question, we return to the governing equation (B7) and compare the magnitudes of terms that were subsequently neglected in the two derivations. For $v_{o} \ll 1,\left(1+v_{o} \mathcal{V}\right)^{-1} \approx\left(1-v_{o} \mathcal{V}\right)$ and to first order the terms neglected in the linearized (expanding) version are

$$
\frac{a D_{c}}{b V_{o} v_{o}}\left(-\dot{v}_{o} \mathcal{V}+v_{o} \mathcal{V} \mathcal{V}^{\prime} \dot{\xi} \dot{L} / L\right)
$$

The neglected elastic term in the localizing solution is

$$
\frac{\mu^{\prime} D_{c}}{2 b \sigma L} \mathcal{H}\left(\mathcal{V}^{\prime}\right)
$$

Because the $\xi$-dependent variables are of order 1 , these expressions are of comparable magnitude when

$$
\frac{\dot{v}_{o}}{v_{o}}+\frac{\dot{L}}{L} \approx \frac{b}{a D_{c}} \frac{V_{o}}{v_{o}} \frac{L_{b}}{2 L}
$$

Using the (linearized) result (B25) to eliminate $\dot{v}_{o} / v_{o}$, this becomes

$$
L \approx L_{b} / 2 v_{o}
$$

[93] If instead of (B25) one uses the no-elasticity results (B12) and (B9) evaluated at $t=0$, the result is the same to within a factor of 1 to 2 (depending upon the magnitude of $\left.v_{o}[0]\right)$. The implication is that for $L \gtrsim L_{b} / v_{o}$ one expects elasticity to be truly negligible and localization to dominate immediately, while for $L \lesssim L_{b} / v_{o}$ elasticity is nonnegligible, even for $L \gg L_{b}$, and expansion should dominate initially. This is confirmed by numerical simulations (that employ $\dot{\theta}=$ $-\Omega$ ) in that simulations with $L(0) / L_{b}=10$ initially undergo expansion for $v_{o}=0.01$ but localization for $v_{o}=1$. Because both $L$ and $v_{o}$ increase with time for simulations that initially expand, eventually $L v_{o}$ exceeds $L_{b}$ and localization is guaranteed. During localization $L v_{o}$ increases (as $v_{o} /\left[1+v_{o}\right]^{1 / 2}$; see equation (B13)), so localization continues until $L \gg L_{b}$ becomes invalid and elasticity again becomes important. 
[94] Because the simulations in Figure 12 employ the full evolution equation $\dot{\theta}=1-\Omega$, the velocity of the expansion $(\dot{L})$ is more rapid than predicted by (B23) and the value of $L v_{o}$ at the transition to localization may, for large $a / b$, be considerably larger than $L_{b}$. We can rationalize this by noting that the initial expansion, and for large $a / b$ even the transition to localization, occur while $\Omega$ is only slightly larger than 1 . This reduces the effective slip-weakening rate without changing the strength of elastic interactions, so it diminishes the tendency for localization relative to that for expansion.

[95] Acknowledgments. We thank Alain Cochard for providing us with notes on the numerical solution of the governing equations and his elastodynamic code used to check our radiation damping solutions, Jim Rice for reminding us what self-similarity really means, and Nadia Lapusta for advice on notation. This paper benefited from comments by two quasianonymous reviewers and the associate editor. Supported by NSF award EAR-0126184.

\section{References}

Abercrombie, R. E. (1996), The magnitude-frequency distribution of earthquakes recorded with deep seismometers at cajon pass, southern california, Tectonophsysics, 261, 1-7.

Ampuero, J.-P. (2002), Etude physique et numérique de la nucléation des séismes, Ph.D. thesis, Univ. Paris 7, Denis Diderot, Paris.

Beeler, N. M., T. E. Tullis, and J. D. Weeks (1994), The roles of time and displacement in the evolution effect in rock friction, Geophys. Res. Lett., 21, 1987-1990

Bizzarri, A., and M. Cocco (2003), Slip-weakening behavior during the propagation of dynamic ruptures obeying rate- and state-dependent friction laws, J. Geophys. Res., 108(B8), 2373, doi:10.1029/2002JB002198.

Blanpied, M. L., C. J. Marone, D. A. Lockner, J. D. Byerlee, and D. P. King (1998), Quantitative measure of the variation in fault rheology due to fluid-rock interactions, J. Geophys. Res., 103, 9691-9712.

Chen, Y. T., and L. Knopoff (1986), Static shear crack with a zone of slipweakening, Geophys. J. R. Astron. Soc., 87, 1005-1024.

Dascalu, C., I. R. Ionescu, and M. Campillo (2000), Fault finiteness and initiation of dynamic shear instability, Earth Planet. Sci. Lett., 177, $163-176$.

Dieterich, J. H. (1979), Modeling of rock friction: 1. Experimental results and constitutive equations, J. Geophys. Res., 84, 2161-2168.

Dieterich, J. H. (1992), Earthquake nucleation on faults with rate- and statedependent friction, Tectonophysics, 211, 115-134.

Dieterich, J. H. (1994), A constitutive law for rate of earthquake production and its application to earthquake clustering, J. Geophys. Res., 99, $2601-$ 2618.

Dieterich, J. H., and B. D. Kilgore (1994), Direct observation of frictional contacts: New insights for state-dependent properties, Pure Appl. Geophys., 143, 283-302.

Dieterich, J. H., and B. D. Kilgore (1996), Implications of fault constitutive properties for earthquake prediction, Proc. Natl. Acad. Sci. U.S.A., 93, $3787-3794$.

Dodge, D. A., G. C. Beroza, and W. L. Ellsworth (1996), Detailed observations of California foreshock sequences: Implications for the earthquake initiation process, J. Geophys. Res., 101, 22,37122,392

Ellsworth, E. L., and G. C. Beroza (1995), Seismic evidence for an earthquake nucleation phase, Science, 268, 851-855.
Ellsworth, E. L., and G. C. Beroza (1998), Observation of the seismic nucleation phase in the Ridgecrest, California, earthquake sequence, Geophys. Res. Lett., 25, 401-404.

Hinch, E. J., and D. G. Crighton (Eds.) (1991), Perturbation Methods, Cambridge Univ. Press, New York.

Kilgore, B. D., M. L. Blanpied, and J. H. Dieterich (1993), Velocity dependent friction of granite over a wide range of conditions, Geophys. Res. Lett., 20, 903-906.

Lapusta, N., and J. R. Rice (2002), Nucleation of rate and state frictional instability under non-uniform loading, Eos Trans. AGU, 83(47), Fall Meet. Suppl., Abstract S61E-05.

Lapusta, N., and J. R. Rice (2003), Nucleation and early seismic propagation of small and large events in a crustal earthquake model, J. Geophys. Res., 108(B4), 2205, doi:10.1029/2001JB000793.

Lawn, B. (1993), Fracture of Brittle Solids, 2nd ed., Cambridge Univ. Press, New York.

Marone, C. (1998), Laboratory-derived friction laws and their application to seismic faulting, Annu. Rev. Earth Planet. Sci., 26, 643-646.

Marone, C., and B. Kilgore (1993), Scaling of the critical slip distance for seismic faulting with shear strain in fault zones, Nature, 362, 618-621.

Nakatani, M. (2001), Conceptual and physical clarification of rate and state friction: Frictional sliding as a thermally activated rheology, J. Geophys. Res., 106, 13,347-13,380.

Press, W. H., B. P. Flannery, S. A. Teukolsky, and W. T. Vetterling (1986), Numerical Recipes: The Art of Scientific Computing, Cambridge Univ. Press, New York.

Ranjith, K., and J. R. Rice (2003), Stability of quasi-static slip in a single degree of freedom elastic system with rate and state dependent friction, J. Mech. Phys. Solids, 47, 1207-1218.

Rice, J. R. (1993), Spatio-temporal complexity of slip on a fault, J. Geophys. Res., 98, 9885-9907.

Rice, J. R., and A. L. Ruina (1983), Stability of steady frictional slipping, J. Appl. Mech., 50, 343-349.

Rice, J. R., and S. T. Tse (1986), Dynamic motion of a single degree of freedom system following a rate- and state-dependent friction law, J. Geophys. Res., 91, 521-530.

Rice, J. R., N. Lapusta, and K. Ranjith (2003), Rate- and state-dependent friction and the stability of sliding between elastically deformable solids, J. Mech. Phys. Sol., 49, 1865-1898.

Ruina, A. (1983), Slip instability and state variable friction laws, J. Geophys. Res., $88,10,359-10,370$.

Scholz, C. H. (1988), The critical slip distance for seismic faulting, Nature, $336,761-763$.

Segall, P., and J. R. Rice (2004), Transition from frictional weakening to shear heating induced thermal pressurization, Eos Trans. AGU, 85(47), Fall Meet. Suppl., Abstract T22A-07.

Spence, D. A., and P. Sharp (1895), Self-similar solutions for elastohydrodynamic cavity flow, Proc. R. Soc. London, Ser. A, 400, 289313.

Suo, Z., M. Ortiz, and A. Needleman (1992), Stability of solids with interfaces, J. Mech. Phys. Solids, 40, 613-640.

Tullis, T. E. (1988), Rock friction constitutive behavior from laboratory experiments and its implications for an earthquake prediction field monitoring program, Pure Appl. Geophys., 126, 555-588.

Uenishi, K., and J. R. Rice (2003), Universal nucleation length for slipweakening rupture instability under nonuniform fault loading, $J$. Geophys. Res., 108(B1), 2042, doi:10.1029/2001JB001681.

J.-P. Ampuero, Institute of Geophysics, Seismology and Geodynamics ETH Honggerberg (HPP), CH-8093 Zurich, Switzerland.

A. M. Rubin, Department of Geosciences, Princeton University, Guyot Hall, Princeton, NJ 08544, USA. (arubin@princeton.edu) 